$\underline{\text { Preprint typeset in JHEP style - HYPER VERSION }}$

hep-th/0510121

UCB-PTH-05/31

LBNL-58946

\title{
Decoding the matrix: Coincident membranes on the plane wave
}

\author{
Raphael Bousso and Aleksey L. Mints \\ Center for Theoretical Physics, Department of Physics \\ University of California, Berkeley, CA 94720-7300, U.S.A. \\ and \\ Lawrence Berkeley National Laboratory, Berkeley, CA 94720-8162, U.S.A. \\ E-mail: bousso@lbl.gov, mints@socrates.berkeley.edu
}

\begin{abstract}
At the core of nonperturbative theories of quantum gravity lies the holographic encoding of bulk data in large matrices. At present this mapping is poorly understood. The plane wave matrix model provides a laboratory for isolating aspects of this problem in a controlled setting.

At large boosts, configurations of concentric membranes become superselection sectors, whose exact spectra are known. From the bulk point of view one expects product states of individual membranes to be contained within the full spectrum. However, for non-BPS states this inclusion relation is obscured by Gauss law constraints. Its validity rests on nontrivial relations in representation theory, which we identify and verify by explicit computation.
\end{abstract}




\section{Contents}

1. Introduction 2

2. The plane wave: vacua, states, and representations 6

2.1 Hamiltonian and vacua 6

2.2 Quantization and the free limit 7

2.3 Spectra and representations 8

2.4 Single membrane 9

2.5 Noncoincident membranes

2.6 General vacuum

3. Coincident membranes 12

3.1 Product spectrum

3.2 Full spectrum

3.3 Assessment 15

4. Irrelevance of the single particle spectrum 16

5. Comparison at finite occupation number 17

5.1 Auxiliary Young diagrams 18

5.2 Interpretation 19

5.3 BPS states 20

5.4 Explicit evaluation and comparison 21

6. Mapping states 23

6.1 Conjugation of auxiliary diagrams 23

6.2 Center of mass ground states 24

7. M-theory limit 25

A. Coefficients of irreducible representations 27

B. Representations and plethysm: Notation, examples, and properties 28

B.1 Young diagrams and tableaux 2

B.2 Schur functions 29

B.3 Plethysm 
G. Trace relations 32

C.1 Algebraic relations 33

C.2 Thermodynamic limit 33

C.3 Exact number of states: A bosonic model 34

C.3.1 Exact number of states of the full spectrum 34

C.3.2 Exact number of states ignoring trace relations

D. Proofs 39

D.1 Distinguishable particles 39

D.2 Mapping states with $U(1)$ spacetime symmetry 40

\section{Introduction}

String theory has succeeded in producing nonperturbative formulations of quantum gravity, such as Matrix theory [1] and the AdS/CFT correspondence [2]. Their application to many problems of physical interest is encumbered by a number of restrictions and technical challenges. Among them is the requirement of an asymptotic region where states are defined; such regions are not expected in a realistic universe. Another limitation is the lack of control over non-supersymmetric excitations; again, this stands in the way of treating problems such as cosmology, the interior of black holes, and spacelike singularities.

However, great difficulties arise already in simpler situations, because of the enormous complexity of the scrambled encoding of physical data in a large matrix. In this paper, we analyze an aspect of the decoding problem, in the setting of the BerensteinMaldacena-Nastase (BMN) matrix model [3].

We work in a free limit where the only nontrivial aspect of the theory lies in the matrix character of the degrees of freedom. This will allow us to focus very sharply on the kinematic problems arising from the complexity of the matrices, without further complications from dynamical evolution. Moreover, it enables us to analyze and compare full spectra, which are known exactly. (As we shall show, the encoding problem we pose simplifies significantly when restricted to BPS states.)

The original Matrix theory [1] was formulated for asymptotically flat spacetime. The understanding of its spectrum is complicated by flat directions. For example, the existence of appropriate ground states has been difficult to demonstrate rigorously, the distinction between single and multiple membranes is problematic, and the description of transverse M5-branes has yet to be understood (see Ref. [4] for a review). 
In the $11 \mathrm{D}$ plane wave [3] the curvature of the flux background acts as a harmonic potential for transverse motion, lifting the flat directions. As a result, the spectrum can be computed exactly in a certain perturbative limit [5]. In the same limit, the number of distinct M2 branes is conserved and becomes a superselection sector, despite the absence of a conserved charge. Protected states [6] within the spectrum provide evidence [7] for the beautiful conjecture [3] that transverse M5 branes arise nonperturbatively as the large $m$ limit of $m$ coincident M2 branes, though the details of this transmutation remain mysterious.

In this paper we study the spectrum of a finite number $m$ of coincident membranes in the plane wave matrix model. We ask whether and how a large matrix encodes the excitations of these $m$ physical constituents. Though we work in the perturbative limit where matrix interactions are suppressed, this question is nontrivial due to gauge constraints. The matrix excitations are singlets under $U(m)$ and correspond to products of traces. Hence, individual operators in the $m \times m$ matrices cannot be excited independently, as would be required for the straightforward generation of the product spectrum.

We compare the spectrum obtained in the full matrix model with the spectrum of a product of the constituent individual membranes. The full spectrum is constructed as follows. One begins with a set of matrix creation operators, which act on the vacuum and form the same representations as the one-particle states. For a general state one assigns up to $m$ matrices ${ }^{1}$ to each trace, and then acts on the vacuum with a product of an arbitrary number of traces.

On the other hand, the product spectrum of the constituent membranes is constructed differently. Beginning with the same single-particle spectrum, one assigns an arbitrary number of creation operators to each membrane, and then takes the product of the $m$ single-membrane spectra.

It is relatively straightforward to demonstrate that both spectra contain the same set of BPS states. But in the limit of a free massive matrix model studied here, the two spectra can be compared in far greater detail because all states are exact.

The symmetry group of this model is given by the supergroup $S U(4 \mid 2)$. Each of the two spectra forms an infinite set of increasingly complex irreducible representations of this group, and each spectrum is completely specified by listing the representations it contains. Thus the assertion that the product spectrum is contained as a subset of the full spectrum can be verified by comparing the multiplicities with which each irreducible representation appears in the two spectra.

\footnotetext{
${ }^{1}$ This avoids overcounting due to trace relations; however, it does so only approximately. Further subtleties arise because matrices inside the trace need not all be the same. They are discussed in the main text and in Appendix 9 .
} 
Let us outline and summarize the rest of this paper. In Sec. 2, we review the plane wave matrix model. In addition to the quartic interactions familiar from flat-space $\mathrm{M}$ (atrix) theory, it has mass terms for all transverse directions (and fermions). It also contains a cubic interaction term that stabilizes fuzzy sphere configurations via a Myers effect.

The vacuum of the matrix model is degenerate. There is a $1 / 2$ BPS ground state for each partition of $N$ into positive integers. It describes a set of concentric fuzzy spheres, which can be viewed as rudimentary M2-branes. The size of each sphere is proportional to the corresponding integer in the partition. The net M2 brane charge vanishes for all configurations, and in general, the potential barrier between vacua is finite. Thus, excitations of the system can change the number of fuzzy spheres. In the large $N$ limit, this describes processes such as two membranes merging into a single larger membrane.

At fixed $N$, the matrix model has an adjustable parameter, $\mu$, corresponding to the steepness of the transverse potential. In the $\mu \rightarrow \infty$ limit the matrix model becomes free; only kinetic and mass terms survive. The barriers between vacua become infinite, so each vacuum now forms the ground state of a superselection sector. In every sector, the Hamiltonian can be diagonalized explicitly, yielding a collection of harmonic oscillators. One obtains a discrete spectrum [5] by applying combinations of a finite set of creation operators.

Thus, in the $\mu \rightarrow \infty$ limit, a given vacuum configuration of fuzzy spheres persists for all excited states. This makes it possible (though not automatic) that the internal states of an individual fuzzy sphere survive unmodified as additional fuzzy spheres are added to the system. If this is the case, then the spectrum should contain all possible products of states associated with the individual oscillations of each constituent. For noncoincident spheres, this is indeed guaranteed by the mutual independence of the operators associated with the diagonal blocks representing each object inside the full matrix.

Next, we turn to the question of whether the same holds true for $m$ coincident spheres. In Sec. 3 we write down formal expressions generating the representations contained in the product spectrum, and, following Ref. [6], those in the full spectrum. They are both given by sums of powers of the same one-particle spectrum, but with different symmetrization patterns applied. As a consequence, the product representations cannot be straightforwardly identified as a subset of the full spectrum, except in a few special cases: if only one membrane is present (which is trivial), or in the limit $m \rightarrow \infty$, which is problematic in the perturbative regime. ${ }^{2}$

\footnotetext{
${ }^{2}$ For a toy matrix model with only one transverse direction, the two spectra would also be trivially
} 
Thus, for all finite $m>1$, the multiplicities of representations must be worked out explicitly, and the results for the two spectra must be compared. Unfortunately, already the common single-particle spectrum underlying both spectra can be arbitrarily rich and depends on the size of the coincident fuzzy spheres. In Sec. 14 we simplify our task by demonstrating that the product spectrum will be a subset of the full spectrum, if the same is true for the much simpler case where one pretends that the symmetry group is $U(\infty)$, and the single particle spectrum is taken to consist only of the fundamental of $U(\infty) .^{3}$

However, even after this simplification one still faces the task of comparing two infinite sets of irreducible representations for each value of $m>1$. In Sec. 5 we break up this task into finite parts by noting that the total occupation number $F$ (the number of operators applied to the vacuum) must be the same for two representations to agree. We find expressions generating only the part of each spectrum corresponding to a given $F$. Moreover, we show that for each sector $F$, it suffices to check only a finite set of values of $m$. The $F$-sector of the full spectrum will contain the representations found in the $F$ sector of the product spectrum if and only if this holds for $2 \leq m \leq F-3$. We describe an algorithm for carrying out this check, and compute the relevant representations for all $F \leq 23$. We find that the results are consistent with the conjectured inclusion relation. (A general proof is left to future work.) We also prove that the number of BPS states matches precisely for every $m$ and $F$.

In Sec. 6, we exhibit some of the nontrivial aspects of the mapping of product states into the full spectrum. We identify on both sides all states without center-ofmass motion. In the full spectrum, this corresponds to the straightforward elimination of certain reps [6]. For the product spectrum, however, it requires taking linear combinations across different copies of a representation. This shows that in the natural bases, a given product representation will generically be mapped into a nontrivial linear combination of equivalent representations in the full spectrum.

In Sec. 7 we discuss subtleties that arise in the M-theory limit, which corresponds to sending $N \rightarrow \infty$ while holding $m$ and the longitudinal momentum fixed. The question then becomes whether M-theory, in the large $\mu$ limit, can describe $m$ noninteracting membranes of equal size.

Various appendices contain additional information; in particular, properties of plethysm are described in Appendix B.

It is interesting that even the relatively simple decoding problem studied here be-

related, because the symmetry group is $U(1)$ (see Sec. 6.2). Hence, the problem cannot be simplified in this manner.

${ }^{3} \mathrm{By} U(\infty)$ we mean $U(p)$ with $p \rightarrow \infty$; the point is just to keep all Young diagrams regardless of the amount of antisymmetrization. 
comes extremely complex, in part due to Gauss law constraints (which we treat exactly) and in part due to the subtleties of trace relations (which we treat only approximately). More powerful techniques than we are aware of may allow a proof of the inclusion relation conjectured in Sec. 1 . With a more appropriate mathematical framework or by mapping to different physical degrees of freedom, one might carry out the comparison of spectra without approximations, and ideally, analytically. Matrix encodings should then become more transparent, and may well teach us lessons about quantum gravity that go beyond the settings of asymptotically flat and AdS spacetime.

\section{The plane wave: vacua, states, and representations}

In this section we review the spectrum of the plane wave matrix model in the $\mu \rightarrow \infty$ limit, and the representations it forms. We follow Refs. $[5,6]$, where more details can be found.

\subsection{Hamiltonian and vacua}

The $U(N)$ plane wave matrix model is given by the Hamiltonian

$$
\begin{array}{r}
H=R \operatorname{Tr}\left(\frac{1}{2} \Pi_{A}^{2}-\frac{1}{4}\left[X_{A}, X_{B}\right]^{2}-\frac{1}{2} \Psi^{\top} \gamma^{A}\left[X_{A}, \Psi\right]\right) \\
+\frac{R}{2} \operatorname{Tr}\left(\left(\frac{\mu}{3 R}\right)^{2} X_{i}^{2}+\left(\frac{\mu}{6 R}\right)^{2} X_{a}^{2}\right. \\
\left.+i \frac{\mu}{4 R} \Psi^{\top} \gamma^{123} \Psi+i \frac{2 \mu}{3 R} \epsilon^{i j k} X_{i} X_{j} X_{k}\right) .
\end{array}
$$

Indices $A \ldots$ run from 1 to $9 ; i \ldots$ run from 1 to 3 ; and $a \ldots$ run from 4 to 9 . This model was proposed [3] to describe M-theory on the maximally supersymmetric plane wave background of eleven-dimensional supergravity,

$$
\begin{aligned}
d s^{2} & =-2 d x^{+} d x^{-}+d x^{A} d x^{A}-\left(\frac{\mu^{2}}{9} x^{i} x_{i}+\frac{\mu^{2}}{36} x^{a} x_{a}\right)\left(d x^{+}\right)^{2}, \\
F_{123+} & =\mu .
\end{aligned}
$$

The M-theory limit is obtained by taking $N \rightarrow \infty$ while holding $N / R$ fixed. In this limit, all nonzero values of the parameter $\mu$ in Eq. (2.1) must be physically equivalent since $\mu$ can be set to unity by a coordinate redefinition in Eq. (2.3), corresponding to a boost in the $x^{ \pm}$plane.

It was further proposed [8] (originally in the context of the flat space model [1]) that the finite $N$ matrices describe the discrete light cone quantization (DLCQ) of Mtheory, in the sector where the center of mass has $N$ units of longitudinal momentum. 
This corresponds to periodically identifying $x^{-} \cong x^{-}+2 \pi R$ in Eq. (2.3). Then there exists a boost-invariant dimensionless quantity, $\mu l_{\mathrm{P}}^{2} / R .{ }^{4}$ Thus, for any finite $N$, the Hamiltonian (2.1) really describes a one-parameter family of matrix models labelled by $\mu .^{5}$

The classical supersymmetric solutions $[3,5]$ of the matrix model are given by $X^{a}=0$ and

$$
X^{i}=\frac{\mu}{3 R} J^{i}
$$

where the $N \times N$ matrices $J^{i}$ form a representation (not necessarily irreducible) of $S U(2)$ :

$$
\left[J^{i}, J^{j}\right]=i \epsilon^{i j k} J^{k}
$$

For any $l$ there is one irreducible $l \times l$ matrix representation of $S U(2)$, so the vacua in question correspond to block-diagonal solutions, one solution for each partition of $N$ into positive integers.

Physically, each block (of size $N_{i}$ ) represents a fuzzy sphere of radius $r \sim \frac{\mu N_{i}}{6 R}$. Thus, a given partition $N=\sum M_{i} N_{i}$ corresponds to a collection of concentric fuzzy spheres. If any of the multiplicities $M_{i}$ is greater than 1 , the collection includes coincident spheres. For example, the partition $N=N$, the "irreducible vacuum", corresponds to a single membrane. The partition $N=1+1+\ldots+1$, the "trivial vacuum", contains $N$ coincident small fuzzy spheres, i.e., $N$ gravitons.

\subsection{Quantization and the free limit}

The theory can be quantized by expanding about the classical vacua

$$
X^{i}=\frac{\mu}{3 R} J^{i}+Y^{i}
$$

and, for convenience, rescaling

$$
Y^{i} \rightarrow \sqrt{\frac{R}{\mu}} Y^{i}, X^{a} \rightarrow \sqrt{\frac{R}{\mu}} X^{a}, t \rightarrow \frac{t}{\mu} .
$$

We denote fermions by $\psi_{I \alpha}$, where $I$ is a fundamental index of $S U(4) \sim S O(6)$, and $\alpha$ is a fundamental index of $S U(2) \sim S O(3)$; see Ref. [5] for the relation between these complex eight-component spinors and the real 16 component spinor $\Psi$.

\footnotetext{
${ }^{4}$ The Planck length is set to unity everywhere else in this paper.

${ }^{5}$ We choose to keep $R$ fixed. However, this is purely conventional. Arguably, it would be conceptually clearer not to introduce the coordinate artifact $\mu$ but to use only $R$ as a parameter.
} 
The action for these fields consists of three separate, $\mu$ - and $R$-independent quadratic actions for $X^{a}, Y^{i}$, and $\psi_{I \alpha}$, respectively; in addition there are cubic and quartic interaction terms. The interaction terms carry prefactors given by positive powers of $R / \mu$. Therefore, matrix interactions are suppressed in the $\mu \rightarrow \infty$ limit.

The precise effective coupling depends on the vacuum [5]. We will be interested mostly in partitions of the form $N=m n$, which represent vacua consisting of $m$ coincident membranes, each with $n$ units of longitudinal momentum. For such vacua the effective coupling behaves like an 't Hooft coupling $\lambda=g^{2} m$, with $g^{2}=\left(\frac{R}{\mu n}\right)^{3}$. But for any partition, the effective coupling can be made arbitrarily small by taking $\mu$ sufficiently large. Moreover, the height of the potential energy barriers separating different vacua will scale with a positive power of $\mu$.

In the limit $\mu \rightarrow \infty$ the theory thus becomes a free massive matrix model. Each vacuum becomes the ground state of a superselection sector and can be quantized separately. Explicit diagonalization converts the Hamiltonian into a collection of free harmonic oscillators. They can be excited independently of one another except where constrained by gauge invariance. Physically, they correspond to effects such as center of mass oscillations in the transverse potential generated by the flux, and internal oscillations of the fuzzy spheres about their spherical ground state. We now turn to a discussion of the resulting spectra.

\subsection{Spectra and representations}

The preceding discussion implies that the spectrum will consist of towers of states of various harmonic oscillators. Hence we can associate to each state a total occupation number, $F$, given by the total number of standard creation operators that are required to create this state from the vacuum. Note that $F$ is not in general proportional to the energy of the state, since different oscillators will have different energy spacing.

All states must fall into representations of the symmetry group of the theory, the supergroup $S U(4 \mid 2)$. The full set of physically admissible representations contains continuous branches, as well as a discrete subset of tensor representations. The tensor representations have integer highest weight and carry only fundamental indices of $S U(4 \mid 2)$. As is familiar from the representation theory of ordinary unitary groups, each irreducible tensor representation is fully characterized by the pattern in which its indices are symmetrized or antisymmetrized, i.e., by a particular Young tableau whose number of boxes agrees with the number of fundamental indices.

Each such "supertableau" subsumes a set of $S U(4) \times S U(2)$ representations, as described in Refs. [6,9]. The energy of an individual $S U(4) \times S U(2)$ representation is 
given by ${ }^{6} n_{4} / 12+n_{2} / 6$, where $n_{4}$ and $n_{2}$ are the number of boxes assigned to the $S U(4)$ and $S U(2)$ indices respectively [6]. Note that because the relevant supersymmetries do not commute with the Hamiltonian, different bosonic representations contained in the same superrepresentation will have different energy. But generally the energy of states associated with a given superrepresentation is of order the number of boxes.

The sum of the irreducible $S U(4 \mid 2)$ representations that are actually present in the spectrum of the theory, for a given partition $P$, will be denoted $R(P) . P$ is specified as a list of nonincreasing integers or a Young diagram. We use the notation $P=$ $\left(N_{1}^{M_{1}} \cdots N_{L}^{M_{L}}\right)$ with $M_{i}, N_{i}$ positive integers and $N_{1}>N_{2}>\ldots>N_{L}$. This describes a Young diagram with $M_{i}$ rows of length $N_{i}$, corresponding to a partition of $N=\sum M_{i} N_{i}$ into integers given by the length of each row. (See Appendix B for further details and notation.) Thus, $R(N)$ refers to the representations of the irreducible vacuum, $R\left(43^{2}\right)$ to the partition of $N=10$ into $4+3+3$, and $R\left(n^{m}\right)$ to the partition of $N=m n$ into $n+n+\ldots+n$.

It was shown in Ref. [6] that at $\mu \rightarrow \infty$, for any partition $P$, the plane wave matrix model contains only tensor representations. This implies that the set $R(P)$ can be specified by an (infinite) list of Young tableaux. Moreover, it can be shown that the generators of $S U(4 \mid 2)$ leave the total excitation number, $F$, invariant. This means that we can break up $R(P)$ into finite chunks according to occupation number. We will denote the (finite) set of representations formed by the states with occupation number $F$ by $R(P)_{F}$.

After diagonalizing the Hamiltonian at $\mu \rightarrow \infty$, one can associate a creation operator to each harmonic oscillator. The full spectrum arises by acting on the vacuum with combinations of these creation operators. Since creation operators commute, ${ }^{7}$ this suggests that the full spectrum will consist of symmetrized products of representations in $R(P)_{1}$, the single particle sector, and that $R(P)$ can be obtained by expanding all such tensor products using the Littlewood-Richardson rule. However, the matrix model is gauged, so physical states must be invariant under $U(N)$ gauge transformations. This Gauss law constraint takes the form

$$
i\left[X^{i}, \Pi^{i}\right]+i\left[X^{a}, \Pi^{a}\right]+2 \psi_{I \alpha} \psi^{\dagger I \alpha}=0
$$

The details of its implementation depends on the vacuum $P$, as we will discuss next.

\subsection{Single membrane}

First, consider the states built on the irreducible vacuum, the partition with a single

\footnotetext{
${ }^{6}$ Due to the rescaling in Eq. (2.7) energies are measured in units of $\mu$.

${ }^{7}$ For operators in representations of a supergroup, this statement subsumes the commutativity of operators in bosonic subgroups and the anticommutativity of the fermionic operators.
} 
element $N$. In this case the specification of the solution completely fixes the gauge. The $N^{2}-1$ nontrivial generators of $U(N)$ gauge transformations show up as a set of zeromode oscillators when the Hamiltonian is diagonalized. These gauge orbit directions are not associated with creation operators. The states obtained from creation operators associated with the remaining sets of oscillators are then automatically physical and gauge-fixed.

The physical creation operators carry an even number of antisymmetrized $S U(4 \mid 2)$ indices $^{8}$

$$
a_{\left[I_{1} I_{2}\right]}, a_{\left[I_{1} I_{2} I_{3} I_{4}\right]}, \ldots, a_{\left[I_{1} \cdots I_{2 N}\right]} .
$$

Hence the single particle states $(F=1)$ form single-column representations of $S U(4 \mid 2)$ with $2,4, \ldots, 2 N$ boxes:

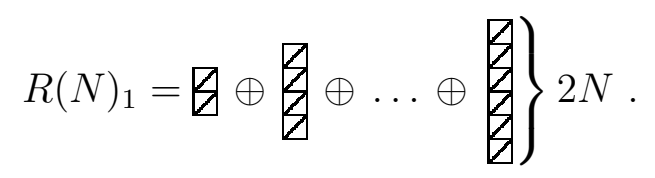

The full spectrum is generated by all combinations of physical creation operators. Since the gauge is already completely fixed, there are no restrictions on such combinations. Hence, the full list of irreps, $R(N)$, is given by

$$
R(N)=\sum_{F} R(N)_{F}=\sum_{F} \operatorname{sym} R(N)_{1}^{F} .
$$

This can also be written in the form

$$
\left.R(N)=\bigoplus_{\left\{n_{i}\right\}}\left[\operatorname{sym}(甘)^{n_{1}} \otimes \operatorname{sym}(\xi)^{n_{2}} \otimes \ldots \otimes \operatorname{sym}(\xi) 2 N\right)^{n_{N}}\right] .
$$

Symmetrization is necessary for powers of identical representations in order to avoid overcounting states, since exchange of the complete set of indices between any two operators in the power leaves the state invariant.

\subsection{Noncoincident membranes}

A partition of $N$ into $L$ different integers $N_{i}$ corresponds to $L$ noncoincident concentric spheres of radii proportional to $N_{i}$. This configuration preserves a portion of the gauge group $U(N)$, namely $U(1)^{L}$ (including the trivial $U(1)$ associated with the center of mass), under which physical states must be invariant.

\footnotetext{
${ }^{8}$ Except for Sec. 6.2 we do not use annihilation operators. Hence we drop the $\dagger$ symbol on creation operators in the interest of a less cluttered notation.
} 
In this configuration the matrices break up into $L^{2}\left(N_{i} \times N_{j}\right)$ blocks, which are separately diagonalized. The $i$-th diagonal block carries the same set of oscillators as the single membrane vacuum with $N=N_{i}$ :

$$
a_{\left[I_{1} I_{2}\right]}^{i i}, a_{\left[I_{1} I_{2} I_{3} I_{4}\right]}^{i i}, \ldots, a_{\left[I_{1} \cdots I_{2 N_{i}}\right]}^{i i} ; i=1, \ldots, L .
$$

Again, the $N_{i}^{2}-1$ zero modes correspond to gauge orbits and are discarded. The remaining operators in the diagonal blocks do not break the $U(1)^{L}$ invariance of the vacuum and hence can be applied without restriction. The resulting spectrum forms precisely the representations in the tensor product $\prod R\left(N_{i}\right)$ of the individual membranes' sets of representations.

Thus, the noncoincident multimembrane vacuum trivially contains the degrees of freedom of its constituents:

$$
R\left(N_{1} \cdots N_{L}\right) \supseteq \prod_{i=1}^{L} R\left(N_{i}\right),
$$

where $R\left(N_{i}\right)$ is given by Eq. (2.11). Indeed, this relation holds at each individual level of the total occupation number, as it should:

$$
R\left(N_{1} \cdots N_{L}\right)_{F} \supseteq\left[\prod_{i=1}^{L} R\left(N_{i}\right)\right]_{F},
$$

where we recall that the final index denotes the restriction of the full spectrum to representations with occupation number $F$.

The full spectrum contains additional representations coming from the off-diagonal blocks, each of which has its own set of oscillators. The associated operators again carry purely antisymmetrized $S U(4 \mid 2)$ indices. The number of indices (and thus the number of boxes in the corresponding single-column representation) ranges in steps of 2 from $\left|N_{i}-N_{j}\right|+2$ to $N_{i}+N_{j}$ :

$$
a_{\left[I_{1} \cdots I_{\left.\left|N_{i}-N_{j}\right|+2\right]}\right.}^{i j}, a_{\left[I_{1} \cdots I_{\left|N_{i}-N_{j}\right|+4}\right]}^{i j}, \ldots, a_{\left[I_{1} \cdots I_{N_{i}+N_{j}}\right]}^{i j} ; i \neq j .
$$

Since the off-diagonal blocks transform in the bifundamental of $U(1)_{i} \times U(1)_{j}$, their operators must be combined to form $U(1)^{L}$ invariants (e.g., $a^{12} a^{23} a^{31}|0\rangle$ ).

These off-diagonal excitations connect pairs of membranes by tubes wrapping the eleventh dimension [10]. In the decompactification limit $\left(N \rightarrow \infty\right.$, keeping $N_{i} / N$ fixed), such tubes become infinitely massive. Indeed, the energy of the lightest off-diagonal state is of order $\left|N_{i}-N_{j}\right|$ and diverges in this limit. 


\subsection{General vacuum}

A general partition $\left(N_{1}^{M_{1}} \cdots N_{L}^{M_{L}}\right)$ corresponds to $L$ concentric stacks of coincident membranes. The $i$-th stack contains $M_{i}$ membranes of size $N_{i}$ sitting on top of one another. The general vacuum state is invariant under $U\left(M_{1}\right) \times \ldots \times U\left(M_{L}\right)$. For physical excitations the same invariance is enforced by the Gauss law constraint. In general, this constraint significantly complicates the explicit construction of the spectrum and especially its comparison with the constituent spectra. We now turn to a detailed analysis of this problem.

We conclude this review section by pointing once more to Refs. [3,5,6], where more detailed descriptions and derivations can be found.

\section{Coincident membranes}

In this section we present the spectra to be compared. We begin by reducing the most general problem to a simpler question.

We would like to know whether the spectrum of a general membrane configuration $\left(N_{1}^{M_{1}} \cdots N_{L}^{M_{L}}\right)$ contains the product states arising from its constituents, i.e., whether a relation analogous to Eq. (2.15) holds in the general case. By arguments completely analogous to those given in Sec. 2.5 for noncoincident membranes, it is trivial to show that

$$
R\left(N_{1}^{M_{1}} \cdots N_{L}^{M_{L}}\right) \supseteq \prod_{i=1}^{L} R\left(N_{i}^{M_{i}}\right),
$$

where $R\left(N_{i}^{M_{i}}\right)$ refers to the spectrum that the $i$-th stack of coincident branes would have by itself. Thus, the independence of the degrees of freedom of one stack of membranes from those of another is manifest.

Then the remaining problem is to identify the individual membranes within a single stack. This requires the comparison of two spectra. The first consists of the symmetrized product states of $m$ identical fuzzy spheres of size $n$. The second is the spectrum $R\left(n^{m}\right)$ built on the matrix theory vacuum corresponding to the partition of $N=m n$ into $m$ coincident fuzzy spheres.

\subsection{Product spectrum}

The product spectrum is the $m$-th tensor power of the single membrane spectrum, restricted to representations compatible with the bosonic exchange symmetry of identical membranes:

$$
R^{\text {prod }}\left(n^{m}\right)=\operatorname{sym} R(n)^{m}=\operatorname{sym}\left[\sum_{g=0}^{\infty} \operatorname{sym} R(n)_{1}^{g}\right]^{m}
$$


where Eq. (2.11) has been used for the second equality.

Using properties of sym described in Appendix B, one can eliminate the trivial representation $g=0$ in exchange for introducing another summation:

$$
R^{\text {prod }}\left(n^{m}\right)=\sum_{f=0}^{m} \operatorname{sym}\left[\sum_{g=1}^{\infty} \operatorname{sym} R(n)_{1}^{g}\right]^{f} .
$$

\subsection{Full spectrum}

We turn to the spectrum of the full matrix model in the partition $\left(n^{m}\right)$. In this vacuum, the matrices break up into $m^{2}$ square blocks, each of which gauge-fixes $n^{2}-1$ of the $U(N)$ generators. The vacuum is invariant under the remaining $U(m)$ gauge group, with $m^{2}-1$ nontrivial generators. The Gauss law constraint, Eq. (2.8), demands that physical states are likewise invariant under $U(m)$.

In each block the Hamiltonian can be diagonalized [5], leading to the set of operators found for the single membrane vacuum with $N=n$. This includes $n^{2}-1$ zero modes corresponding to gauge orbits, which are tossed out. Combining all blocks, one can think of each physical operator as a $m \times m$ matrix operator, e.g.,

$$
A_{\left[I_{1} I_{2}\right]}=\left(\begin{array}{cccc}
a_{\left[I_{1} I_{2}\right]}^{11} & a_{\left[I_{1} I_{2}\right]}^{12} & \ldots & a_{\left[I_{1} I_{2}\right]}^{1 m} \\
a_{\left[I_{1} I_{2}\right]}^{21} & a_{\left[I_{1} I_{2}\right]}^{22} & \ldots & a_{\left[I_{1} I_{2}\right]}^{2 m} \\
\vdots & \vdots & \ddots & \vdots \\
a_{\left[I_{1} I_{2}\right]}^{m 1} & a_{\left[I_{1} I_{2}\right]}^{m 2} & \ldots & a_{\left[I_{1} I_{2}\right]}^{m m}
\end{array}\right) .
$$

$U(m)$ invariant objects arise from traces of products of these matrices. Hence, physical states are obtained by acting on the vacuum with products of traces of products of matrix creation operators, e.g.:

$$
\operatorname{Tr}\left(A_{\left[I_{1} I_{2}\right]} A_{\left[J_{1} \cdots J_{6}\right]}\right) \operatorname{Tr}\left(A_{\left[K_{1} \cdots K_{8}\right]}\right)|0\rangle .
$$

Next we discuss the $S U(4 \mid 2)$ representations formed by the physical states [6]. Each matrix $A$ is a single column representation since the indices are totally antisymmetrized. This is unaffected by taking the trace, so the single particle states, $\operatorname{Tr} A|0\rangle$, form the same representations as those of a single membrane with $N=n$ :

$$
R\left(n^{m}\right)_{1}=R(n)_{1}
$$

(The supertableaux in $R(n)_{1}$ are shown in Eq. 2.10.)

The full spectrum will be given by tensor products of these representations, subject to appropriate symmetrization. Matrices within a single trace do not commute, so 
total symmetrization would be too restrictive. Instead, the allowed representations must follow a symmetrization pattern, denoted cyc, that is compatible with the cyclic symmetry of the trace. Its properties are described in greater detail in Appendix B, where it is shown that cyc corresponds to the sum of all plethysms by Young diagrams with an odd number of rows. ${ }^{9}$ In particular, the representations generated by cyc always contain those generated by sym as a subset.

This suggests that single trace states with $g$ operators will form representations cyc $R(n)_{1}^{g}$. However, there is a danger of overcounting due to trace relations. Traces containing more than $m$ equal factors are equal to products of smaller traces. Correspondingly we shall require $g \leq m$, leading to single-trace representations

$$
R_{\text {single-trace }}^{\prime}\left(n^{m}\right)=\sum_{g=1}^{m} \text { cyc } R(n)_{1}^{g} .
$$

A prime has been added to indicate that $R_{\text {single-trace }}^{\prime}$ is a truncated spectrum. This is because our restriction $g \leq m$ is actually somewhat excessive. There are many different irreducible representations, and each contains several different matrix operators. The standard trace relations apply rigorously only to traces of a power of a single operator. Indeed, independent states with more than $m$ operators inside the trace can exist if not all the operators are equal. However, it is cumbersome ${ }^{10}$ to extract the allowed combinations. Hence, though we only wish to show that the product of individual membrane spectra is contained in the full spectrum $R\left(n^{m}\right)$, we will in fact argue that the stronger statement is true that it is contained already among the representations computed from the truncated single-trace spectrum $R_{\text {single-trace }}^{\prime}$

A general state contains multiple traces. These factors commute and must be symmetrized. The full spectrum (up to the truncation introduced earlier) is thus

$$
\begin{aligned}
R^{\prime}\left(n^{m}\right) & =\sum_{f=0}^{\infty} \operatorname{sym}\left[R_{\text {single-trace }}^{\prime}\left(n^{m}\right)\right]^{f} \\
& =\sum_{f=0}^{\infty} \operatorname{sym}\left[\sum_{g=1}^{m} \text { cyc } R(n)_{1}^{g}\right]^{f},
\end{aligned}
$$

assuming that no trace relations arise for multitrace states. As explained in Appendix C, one expects this assumption to be well satisfied for occupation numbers

\footnotetext{
${ }^{9}$ We thank M. Van Raamsdonk for pointing this out to us.

${ }^{10}$ See the rather intricate analysis for even the simple case $n=1, m=2, F=3$ in Ref. [6]. The single trace spectrum in this case is given by the plethysm $R(1)^{\otimes\left(1^{3}\right)}$ [which could be denoted loosely as (cyc - sym $) R(1)^{3}$ ]. Moreover, a general state is not allowed to contain more than one such trace. (There is a minor misleading statement in Ref. [6] suggesting that the full cyc is present; note also that the $U(1)$ degrees of freedom are suppressed there.)
} 
$F \ll \operatorname{dim} R(n)_{1} m^{2}$. Note that this upper bound grows rapidly with $n$ and diverges in the M-theory limit. Nevertheless, it is important to keep in mind that our expression for $R^{\prime}$ should be expected to contain a small number of redundant representations. It is possible that eliminating these states could spoil the inclusion relation we find in Sec. 5. In light of the evidence presented in Appendix $\square$ we consider this possibility remote.

\subsection{Assessment}

We would like to determine whether the representations formed by the product spectrum of $m$ membranes, Eq. (3.3), is a subset of the representations formed by the full spectrum of the $U(m)$ theory, Eq. (3.9), as one would expect physically; i.e., is

$$
R^{\prime}\left(n^{m}\right) \supseteq R^{\text {prod }}\left(n^{m}\right) ?
$$

The two expressions given above differ in two ways. The first is quite trivial: as explained in Appendix B, a cyclically symmetrized power (which arises for $U(m)$ ) always contains the corresponding totally symmetrized power (arising in the product spectrum) as a subset. One can thus consider truncating the $U(m)$ spectrum further by replacing the cyc with sym in Eq. (3.9) ${ }^{11}$ This yields the spectrum

$$
R^{\prime \prime}\left(n^{m}\right)=\sum_{f=0}^{\infty} \operatorname{sym}\left[\sum_{g=1}^{m} \operatorname{sym} R(n)_{1}^{g}\right]^{f} \text {. }
$$

The spectra obey $R \supseteq R^{\prime} \supseteq R^{\prime \prime}$. It would be nice to continue this sequence of inclusions to $R^{\text {prod }}$. However, even after replacing cyclic with total symmetrization, a crucial difference between $R^{\prime \prime}$ and $R^{\text {prod }}$ remains: the exchange of the upper limits, $m$ and $\infty$, of the double sum.

Clearly the spectra $R^{\prime \prime}$ and $R^{\text {prod }}$ agree in the limit of infinitely many membranes, $m \rightarrow \infty .{ }^{12}$ Also, one easily verifies that they reduce to the same expression for the case of a single membrane, $m=1$. But for all other values of $m$ the spectra $R^{\prime \prime}$ and $R^{\text {prod }}$ differ, and it is not apparent whether the former contains the latter. Hence a more detailed analysis is needed, which we begin in the next section.

\footnotetext{
${ }^{11}$ This modification includes as a special consequence the earlier truncation $R \rightarrow R^{\prime}$ introduced in Eq. (3.7), since the usual trace relations do apply to traces of totally symmetrized products.

${ }^{12}$ In order to take this limit while keeping the coupling $\lambda$ small and fixed, $\mu$ must be scaled like $m^{1 / 3}$ (see Sec. 2.2).
} 


\section{Irrelevance of the single particle spectrum}

It may appear that the validity of Eq. (3.10) depends on the detailed structure of $R(n)_{1}$, the single-particle spectrum. It contains various irreducible tensor representations of the supergroup $S U(4 \mid 2)$ (see Eq. (2.10)). In particular, in the M-theory $(n \rightarrow \infty)$ limit, $R(n)_{1}$ contains an arbitrarily large number of different irreducible terms. This would appear to complicate the comparison of the spectra $R^{\prime}$ and $R^{\text {prod }}$, which are constructed from symmetrized powers of $R(n)_{1}$. Moreover, it might seem that the validity of Eq. (3.10) could depend on the value of $n$.

However, in this section we will show the problem simplifies considerably: it suffices to show that $R^{\prime} \supseteq R^{\text {prod }}$ for the case where $R(n)_{1}$ is assumed to contain only a single, fundamental representation of the unitary group, which we shall think of as having infinite degree, $U(\infty)$. (Note that this does not even correspond to any choice of parameters for the matrix model at hand.) In particular, we are guaranteed that Eq. (3.10) holds for all $n$ and in the M-theory limit, if this simpler case can be proven.

This simplification arises as follows. Beginning with $R^{\prime}$, write the symmetric and cyclic powers in Eq. (3.9) as plethysms (see Appendix B):

$$
R^{\prime}\left(n^{m}\right)=\sum_{f=0}^{\infty}\left[\sum_{g=1}^{m} R(n)_{1}^{\otimes\left(\text { oddrow }_{g}\right)}\right]^{\otimes(f)},
$$

where the partition $(f)$ denotes the Young diagram corresponding to complete sym-

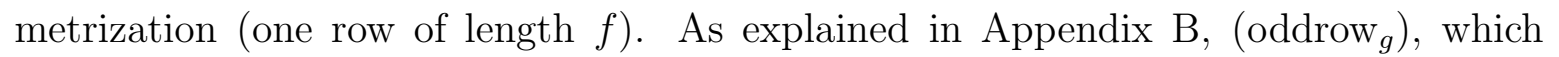
denotes the sum of all Young diagrams with $g$ boxes and an odd number of rows, is the plethysm corresponding to a cyclically symmetrized power.

Since plethysm is distributive in the "exponent", we have

$$
R^{\prime}\left(n^{m}\right)=\left[R(n)_{1}^{\otimes \sum_{g=1}^{m}\left(\text { oddrow }_{g}\right)}\right]^{\otimes \sum_{f=0}^{\infty}(f)} .
$$

Associativity of plethysm implies

$$
R^{\prime}\left(n^{m}\right)=R(n)_{1}^{\otimes}\left[\left(\sum_{g=1}^{m}\left(\text { oddrow }_{g}\right)\right)^{\otimes \sum_{f=0}^{\infty}(f)}\right] .
$$

Similarly, $R^{\text {prod }}$ can be written in the form

$$
R^{\operatorname{prod}}\left(n^{m}\right)=R(n)_{1}^{\otimes\left[\left(\sum_{g=1}^{\infty}(g)\right)^{\otimes \sum_{f=0}^{m}(f)}\right]} .
$$

Hence Eq. (3.10) will hold, irrespective of $R(n)_{1}$, if the corresponding relation holds for the $U(\infty)$ representations contained in the exponents. 
Thus, the problem reduces to the question of whether

$$
S^{\prime}(m) \supseteq S^{\text {prod }}(m)
$$

where

$$
\begin{aligned}
S^{\prime}(m) & \equiv\left(\sum_{g=1}^{m}\left(\operatorname{oddrow~}_{g}\right)\right)^{\otimes \sum_{f=0}^{\infty}(f)}, \\
S^{\operatorname{prod}}(m) & \equiv\left(\sum_{g=1}^{\infty}(g)\right)^{\otimes \sum_{f=0}^{m}(f)} .
\end{aligned}
$$

Unlike $R^{\prime}$ and $R^{\text {prod }}$, these expressions are independent of $n$; the argument $m$ here is not a partition but denotes the number of (arbitrary-size) coincident membranes in the stack.

The $S^{\prime}$ and $S^{\text {prod }}$ coincide with $R^{\prime}$ and $R^{\text {prod }}$ precisely if we set $R(n)_{1}=\square$, as advertised above. This is because sym $\square^{g}$ gives precisely the one-row Young diagram of length $g$, here denoted $(g)$; and cyc $\square^{g}$ gives the odd-row Young diagrams with $g$ boxes. In this sense we have reduced the problem to the simple case where the single particle states lie only in the fundamental of $U(\infty)$.

A similar simplification can be derived involving $R^{\prime \prime}$, the spectrum obtained from the truncation cyc $\rightarrow$ sym. Writing $R^{\prime \prime}$ as a plethysm of $R(n)_{1}$, one obtains $R^{\prime \prime}\left(n^{m}\right)=$ $R(n)_{1}^{\otimes S^{\prime \prime}(m)}$, where

$$
S^{\prime \prime}(m) \equiv\left[\sum_{g=1}^{m}(g)\right]^{\otimes \sum_{f=0}^{\infty}(f)} .
$$

Clearly, Eq. (3.10) follows if it can be shown that $R^{\prime \prime}\left(n^{m}\right) \supseteq R^{\text {prod }}\left(n^{m}\right)$, which in turn is implied by

$$
S^{\prime \prime}(m) \supseteq S^{\text {prod }}(m)
$$

We will provide some evidence below that this relation does hold.

\section{Comparison at finite occupation number}

In the absence of a proof of Eq. (4.9), we would like to assess its plausibility by verifying parts of the relation explicitly. This can be done systematically by restricting both $S^{\prime \prime}(m)$ and $S^{\text {prod }}(m)$ to states with a given occupation number $F$. The occupation number of an irreducible $U(\infty)$ representation in $S^{\prime \prime}$ or $S^{\text {prod }}$ is the number of boxes 
in the corresponding $U(\infty)$ Young diagram. ${ }^{13}$ Clearly, the inclusion relation Eq. (4.9) can only be true if it holds separately for each $F$ :

$$
S^{\prime \prime}(m)_{F} \supseteq S^{\operatorname{prod}}(m)_{F}
$$

\subsection{Auxiliary Young diagrams}

Let us expand

$$
\begin{aligned}
S^{\prime \prime}(m)_{F} & =\bigoplus_{P(F)} c^{\prime \prime}(m)_{P(F)} P(F), \\
S^{\operatorname{prod}}(m)_{F} & =\bigoplus_{P(F)} c^{\operatorname{prod}}(m)_{P(F)} P(F),
\end{aligned}
$$

where the sum is over all partitions of $F$, i.e., all Young diagrams with $F$ boxes. Thus, at finite $F$ we would like to verify that the multiplicities of each representation obey

$$
c^{\prime \prime}(m)_{P(F)} \geq c^{\operatorname{prod}}(m)_{P(F)}
$$

for all $P(F)$. This requires a systematic way of isolating the terms in $S^{\prime \prime}$ and $S^{\text {prod }}$ that lead to $F$-box representations. Then the multiplicities can be tallied up and compared.

Consider the example $m=3$. One can write the expressions for $S$ in the form

$$
\begin{aligned}
& S^{\operatorname{prod}}(3)=\quad \bullet \\
& \oplus \quad(\square \oplus \square \oplus \square \oplus \square \square \oplus \cdots) \\
& \oplus \operatorname{sym}(\square \oplus \square \oplus \square \text { पाप } \oplus \oplus \cdots)^{2} \\
& \oplus \operatorname{sym}(\square \oplus \square \oplus \square \oplus \oplus \square \oplus \cdots)^{3} \text {, }
\end{aligned}
$$

where • stands for the trivial representation, i.e., the vacuum. The first and second row need no evaluation. For the higher powers, the sym ensures that each (commuting) product of representations occurs with multiplicity 1. For example the third row evaluates to

$$
\operatorname{sym} \square^{2} \oplus(\square \cdot \square) \oplus \operatorname{sym} \square^{2} \oplus(\square \cdot \square) \oplus(\square \cdot \square) \oplus \operatorname{sym} \square^{2} \oplus \cdots
$$

Imagine writing the factors in each term underneath one another. This yields a set of "auxiliary Young diagrams". They do not themselves correspond to representations

\footnotetext{
${ }^{13}$ Acting as a plethysm, a representation with $U(\infty)$ occupation number $F$ produces a subset of the $F$-th power of the single particle states $R(n)_{1}$. Hence, the resulting occupation number in the $R$ spectra will again be $F$. This will become quite explicit in the examples below.
} 
in the expansion of Eq. (5.4), but they help us keep track of the terms that will appear there. For example the terms in Eq. (5.5) are in one-to-one correspondence with auxiliary diagrams

$$
\boxminus, \square, \boxplus, \boxminus, \boxplus, \boxplus, \cdots
$$

Note that every Young diagram with 2 rows occurs precisely once. Similarly, the 3factor product terms arising from the last row of Eq. (5.4) are in correspondence with all 3-row auxiliary Young diagrams.

Let us summarize and generalize the above. Expanding the powers of the infinite sum in $S^{\text {prod }}(m)$ into a sum over products, one finds that the resulting terms are in one-to-one correspondence with all auxiliary Young diagrams with up to $m$ rows.

For example the auxiliary diagram ${ }^{1}$ (which will be considered only if $m \geq 3$ ) corresponds to the term $\square \cdot$ sym $\square^{2}$. Further evaluation of this product yields a sum of various irreducible $U(\infty)$ representations, each with $F=4+2+2=8$ boxes.

In order to obtain the multiplicities for all $F$-box representations in $S^{\text {prod }}(m)$, one can thus proceed as follows. Write down all F-box auxiliary Young diagrams with up to $m$ rows; for each, evaluate the symmetric product of the rows; sum up the results. In other words:

$$
S^{\text {prod }}(m)_{F}=\bigoplus_{P(F / m)=\left(q_{1}^{p_{1}} \ldots q_{l}^{p_{l}}\right)} \prod_{i=1}^{l} \operatorname{sym}\left(q_{i}\right)^{p_{i}}
$$

where the sum is over all partitions $P(F / m)$ of $F$ with up to $m$ terms (i.e., $\sum_{i=1}^{l} p_{i} \leq$ $m)$.

For $S^{\prime \prime}(m)$, an analogous argument leads to the following prescription: Write down all F-box auxiliary Young diagrams with up to $m$ columns; for each, evaluate the symmetric product of the rows; sum up the results:

$$
S^{\prime \prime}(m)_{F}=\bigoplus_{P(F \backslash m)=\left(q_{1}^{\left.p_{1} \ldots q_{l}^{p_{l}}\right)}\right.} \prod_{i=1}^{l} \mathrm{sym}\left(q_{i}\right)^{p_{i}},
$$

where the sum is over all partitions $P(F \backslash m)$ of $F$ with no term greater than $m$ (i.e., $\left.q_{1} \leq m\right)$. This will produce all $F$-box representations and their multiplicities $c^{\prime \prime}(m)_{P(F)}$.

\subsection{Interpretation}

The above expressions, Eqs. (5.7) and (5.8) have a straightforward combinatorial interpretation, even in the original context of the $R$-spectra.

The expression for $S^{\text {prod }}(m)_{F}$ corresponds to the problem of distributing $F$ creation operators, each in the fundamental of $U(\infty)$, among $m$ identical objects. The distribution is described first by the partitions of $F$ with no more than $m$ terms $\left(\oplus_{P(F / m)}\right)$. 
For each partition, there are many different states corresponding to a choice of specific operators from the fundamental multiplet. However, the resulting representation is not simply $\square^{F}$. The $q_{i}$ operators assigned to one particle commute and must be totally symmetrized. Thus the resulting states lie in the representation sym $\square^{q_{i}}=\left(q_{i}\right)$ (the one-row diagram with $q_{i}$ ) boxes. The representations for the different particles must be tensored together $\left(\prod_{i=1}^{l}\right)$, taking care to symmetrize between particles that are not distinguished by different oscillator number $\left(\operatorname{sym}\left(q_{i}\right)^{p_{i}}\right)$.

The expression for $S^{\prime \prime}(m)_{F}$ can be similarly understood. In fact, consider the more general case of

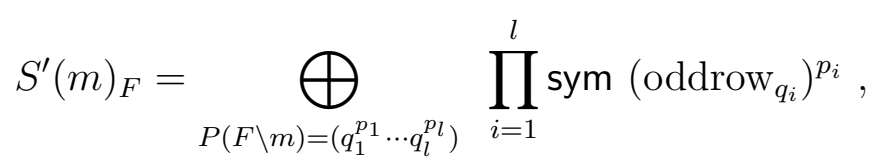

which arises analogous to Eq. (5.8) by restricting Eq. (4.6) to the sector with occupation number $F$. This describes the distribution of $F$ matrix operators among an arbitrary number of traces, assigning no more than $m$ operators to each trace $\left(\oplus_{P(F \backslash m)}\right)$. The operators assigned to one trace are cyclically symmetric. The resulting states lie in cyc $\square^{q_{i}}=\left(\right.$ oddrow $\left._{q_{i}}\right)$. Different traces commute and must be symmetrized. $\left(S^{\prime \prime}(m)_{F}\right.$ is the truncation of this spectrum to those states which are totally symmetrized within each trace.)

With this interpretation, it is clear that these expressions can be lifted back up to the original case where the single-particle representation is more complicated. One replaces the fundamental of $U(\infty)$ with the actual single-particle representation of the symmetry group:

$$
\begin{gathered}
R^{\prime}\left(n^{m}\right)_{F}=\bigoplus_{P(F \backslash m)=\left(q_{1}^{\left.p_{1} \ldots q_{l}^{p_{l}}\right)}\right.} \prod_{i=1}^{l} \operatorname{sym}\left(\operatorname{cyc} R(n)_{1}^{q_{i}}\right)^{p_{i}} ; \\
R^{\prime \prime}\left(n^{m}\right)_{F}= \\
\bigoplus_{P(F \backslash m)=\left(q_{1}^{p_{1} \ldots} q_{l}^{p_{l}}\right)} \prod_{i=1}^{l} \operatorname{sym}\left(\operatorname{sym} R(n)_{1}^{q_{i}}\right)^{p_{i}} ; \\
R^{\operatorname{prod}}\left(n^{m}\right)_{F}=\bigoplus_{P(F / m)=\left(q_{1}^{\left.p_{1} \ldots q_{l}^{p_{l}}\right)}\right.} \prod_{i=1}^{l} \operatorname{sym}\left(\operatorname{sym} R(n)_{1}^{q_{i}}\right)^{p_{i}} .
\end{gathered}
$$

\subsection{BPS states}

There is an important subset of states which are protected as $\mu$ is decreased away from the $\mu \rightarrow \infty$ limit. These "doubly atypical" states are analogues of BPS states [6], and they come from superrepresentations with exactly two equal rows of arbitrary length:

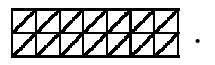


Such representations can only be generated from plethysms of $\square$; no other diagrams in $R(n)_{1}$ (see Eq. (2.10)) will contribute.

In Eqs. (5.10)-(5.12) we have expressed the full and the product spectra as sums over auxiliary diagrams. Each of the three sums contains the same number of auxiliary diagrams (even though they are not necessarily the same diagrams). One finds that each auxiliary diagram contributes precisely one doubly atypical superrepresentation (with $F$ columns). Thus all three spectra contain the same number of doubly atypical states. In other words, the BPS part of the spectra matches exactly.

\subsection{Explicit evaluation and comparison}

We now turn to checking whether the inclusion relation Eq. (5.1) holds for the complete spectra (not just the BPS states). As discussed in Sec. 1 , it will suffice to compare $S^{\prime \prime}(m)_{F}$ and $S^{\text {prod }}(m)_{F}$. Note that the two spectra differ solely in whether $m$ limits the number of columns, or rows, of the auxiliary diagrams. If the diagrams contain fewer than $m$ boxes $(F \leq m)$, neither of these restrictions actually eliminate any diagrams. It follows immediately that the spectra agree identically as long as the total excitation number does not exceed the number of membranes:

$$
S^{\prime \prime}(m)_{F}=S^{\operatorname{prod}}(m)_{F} \text { for all } F \leq m .
$$

This is important because it means that to verify Eq. (4.9) for all $m$ at fixed $F$, it suffices to check only a finite set of spectra $(1 \leq m \leq F-1) .{ }^{14}$

We have used the LiE package [11] to expand both $S^{\prime \prime}(m)_{F}$ and $S^{\text {prod }}(m)_{F}$ into a sum of irreducible representations, as in Eq. (5.2), for all values of the total occupation number $F$ up to 23. We found that Eq. (5.3) holds. This shows that $S^{\prime \prime}(m)_{F} \supseteq$ $S^{\text {prod }}(m)_{F}$ for $F \leq 23$ and all $m$. This is evidence that $S^{\prime \prime} \supseteq S^{\text {prod }}$ for all $m$, as we expect physically. It would be nice to prove this rigorously.

The coefficients $c(m)_{F}$ are listed in Appendix A. However, since we have not been able to prove our result generally, it may be instructive to go through a few examples quite explicitly. This will illustrate the nature of the evidence we provide, and what remains to be proven.

Let us begin with $F=1$. This case is trivial for any $m \geq 1$ : on both sides, there is only one auxiliary Young diagram, $\square$, leading to the same $U(\infty)$ representation, $\square$. For $F=2, m=1$, there is again one allowed Young diagram on each side: $\boxminus$ for $S^{\prime \prime}$, and $\square$ for $S^{\text {prod }}$. Hence, $S^{\prime \prime}(1)_{2}=$ sym $\square^{2}=\square=S^{\text {prod }}(1)_{2}$. For any $m \geq 2$, each side contains the same two auxiliary diagrams: $\square, \Theta$.

\footnotetext{
${ }^{14}$ In fact it suffices to consider $2 \leq m \leq F-3$, which follows from the trivial agreement of the $m=1$ spectra and by analyzing in detail the cancellation of restricted partitions $P(F / m)$ against $P(F \backslash m)$ for $m=F-2, \mathrm{~m}=F-1$, using Eqs. (D.3) and (D.4) below.
} 
Next consider $F=3$. For $m=1$ the auxiliary diagrams are 日 $\left(S^{\prime \prime}\right)$ and $\left(S^{\text {prod }}\right)$. Thus $S^{\prime \prime}(1)_{3}=\square=S^{\text {prod }}(1)_{3}$. The reader will have noticed that independently of $F$, at $m=1$ each side contains only one diagram, and that both lead to one copy of the totally symmetrized $U(\infty)$ representation. This is as it should: at $m=1$ the spectra $S^{\prime}, S^{\prime \prime}$, and $S^{\text {prod }}$ must all agree, since they describe the same object: a single membrane. Hence we will no longer consider $m=1$ below. At $F=3, m=2$, the same new auxiliary diagram appears on both sides $(\boxplus)$, and the agreement persists for larger $m$ according to Eq. (5.14).

Thus, up to occupation number 3 the two spectra agree exactly for all values of $m$ :

$$
S^{\prime \prime}(m)_{F}=S^{\mathrm{prod}}(m)_{F}, \quad F=1,2,3 .
$$

The first difference arises at $F=4$. At $m=1$ one has the usual identity, but $m=2$ permits the following additional auxiliary diagrams:

$$
\text { 曰, 田 }
$$

for $S^{\prime \prime}$ and

$$
\text { 巴, 田 }
$$

for $S^{\text {prod }}$. One can cancel the $\boxplus$ diagram since it will contribute the same irreducible representations to both spectra. The $巴$ diagram yields

$$
\text { प्य } \oplus \text { प् }
$$

which is a proper superset of the representation

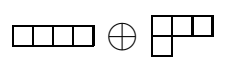

coming from the 1 auxiliary diagram. For any $m>2$ one finds again that the two spectra agree exactly.

Finally, let us go through the $F=5$ case. In the following table, we show for each value of $m$ (first column) the auxiliary diagrams relevant for the full spectrum (second column) and the representations they generate (third column) and similarly for the product spectrum (fourth and fifth columns). The sixth column shows the running difference between the previous two for each $m$; note that it always positive as required for the inclusion relation (3.10) to hold. In Appendix A, we continue the comparison for $6 \leq F \leq 10$. 


\begin{tabular}{|c|c|c|c|c|c|}
\hline$m$ & Aux Full & $S^{\prime \prime}(m)_{F}$ & Aux Prod & $S^{\operatorname{prod}}(m)_{F}$ & $S^{\prime \prime}-S^{\text {prod }}$ \\
\hline 1 & 日 & sym $\square^{5}=\square$ & מחדם & sym $\square^{5}=$ & 0 \\
\hline \multirow[t]{2}{*}{2} & $\bar{\theta}$ & 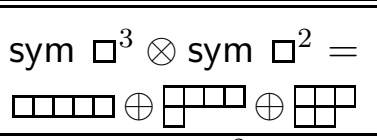 & पण & $\begin{array}{l}\text { sym } \square^{4} \otimes \square= \\
\square \square \oplus \square^{\square} \oplus=\end{array}$ & \multirow[b]{2}{*}{$\boxplus \oplus \bigoplus$} \\
\hline & $\bigoplus$ & $\begin{array}{l}\operatorname{sym}\left(\operatorname{sym} \square^{2}\right)^{2} \otimes \square= \\
\square \square \oplus \oplus \nabla^{\square} \oplus \\
\square \oplus \boxminus\end{array}$ & $\boxplus$ & $\begin{array}{l}\text { sym } \square^{3} \otimes \text { sym } \square^{2}= \\
\square \square \square \oplus \bigoplus \sqcap \oplus \bigoplus\end{array}$ & \\
\hline \multirow[t]{2}{*}{3} & $\overline{\theta+1}$ & 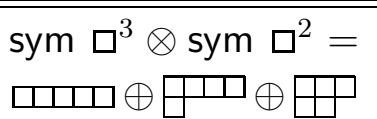 & 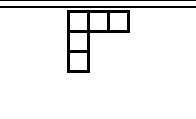 & $\begin{array}{l}\text { sym } \square^{3} \otimes \text { sym } \square^{2}= \\
\square \square \square \oplus{ }^{\prime \prime} \oplus \emptyset\end{array}$ & \multirow[b]{2}{*}{$\boxplus$} \\
\hline & $\uplus$ & $\begin{array}{l}\text { sym } \square^{3} \otimes \text { sym } \square^{2}= \\
\square \text { पाम } \oplus \oplus \emptyset \square\end{array}$ & $\bigoplus$ & 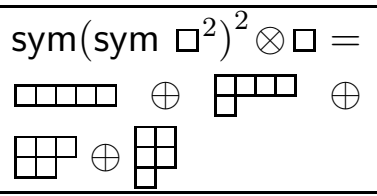 & \\
\hline 4 & $\square^{\square}$ & $\begin{array}{l}\text { sym } \square^{4} \otimes \square= \\
\square \square \oplus \theta \square\end{array}$ & $\bar{\theta}$ & $\begin{array}{l}\text { sym } \square^{2} \otimes \text { sym } \square^{3}= \\
\square \square \oplus \square^{\square} \oplus \bigoplus\end{array}$ & 0 \\
\hline 5 & מחד & sym $\square^{5}=$ & $\overline{\mathrm{B}}$ & sym $\square^{5}=$ & 0 \\
\hline
\end{tabular}

\section{Mapping states}

\subsection{Conjugation of auxiliary diagrams}

In the previous section we have found evidence that the full spectrum of $m$ coincident membranes contains all representations expected from the product states of the individual membranes (and more). However, we only counted multiplicities of representations; we did not ask how a given product state should be mapped into a particular state in the full spectrum. In this section we will learn about some aspects of this mapping. For simplicity, we take $n=1$; then the single particle spectrum contains only one irreducible representation of the symmetry group.

It is instructive to begin by considering an oversimplified case. Let us suppose that the symmetry group is $U(1)$ and the one-particle spectrum consists only of the fundamental representation of $U(1)$. (This would correspond to a bosonic model with only one transverse direction of oscillation.) In this case every auxiliary Young diagram with $g$ boxes leads to the same representation: the totally symmetrized representation with $g$ indices, containing only one state. The product and full spectra then match 
exactly, since they contain the same number of auxiliary diagrams. ${ }^{15}$

One might expect that for more general representations it should also be possible to find a mapping of entire auxiliary Young diagrams of the product spectrum into auxiliary Young diagrams of the full spectrum. The only natural way to do this would be conjugation, the map that flips the Young diagram about its diagonal axis.

However, explicit counterexamples show that this naive mapping cannot be correct. For example, the auxiliary diagram $\bigoplus$ in the $F=10, m=3$ product spectrum contains $U(\infty)$ representations which are not generated by the $\bigoplus$ diagram of the full spectrum. It is only after summing over all allowed auxiliary diagrams at each value of $m$ that the full spectrum becomes a superset of the product spectrum. This suggests that the true map is quite scrambled.

Interestingly, an inclusion relation does hold at the level of individual auxiliary diagrams between products of distinguishable particles and traces. This is proven in Appendix D.1.

\subsection{Center of mass ground states}

One can learn more about the details of the mapping by considering states in which the center of mass degree of freedom is not excited ("c.o.m. ground states" for short). It is straightforward to construct all such states in the full spectrum [6]. Let us denote the $m \times m$ matrix degrees of freedom (such as $X, Y$, and $\psi$ in the $S U(4 \mid 2)$ model) collectively by $A$, and define

$$
B=A-\frac{\operatorname{Tr} A}{m} \mathbf{1}
$$

The trace of $B$ vanishes but traces of higher powers of $B$ (up to $m$ ) are nonzero and mutually independent.

Using $B$ instead of $A$, c.o.m. ground states are constructed as in Sec. 5, except that there will be no contributions from traces of only one matrix. This corresponds to requiring that the first and second column of the auxiliary Young diagram have the same height. All other diagrams vanish because they contain powers of $\operatorname{Tr} B=0$.

On the other hand, product states without center of mass excitations are annihilated by all center of mass annihilation operators:

$$
a_{\mathrm{com}}|\psi\rangle=0
$$

where $a_{\text {com }}=m^{-1 / 2} \sum_{i=1}^{m} a_{i}$. Here the index $i$ refers to the $i$-th "membrane", and Eq. (6.2) must hold for every operator in the single-particle multiplet.

\footnotetext{
${ }^{15}$ The same mapping works for the BPS-protected states $[5,6]$ in the real plane wave matrix model (see Sec. 5.3). In both cases, there is only one relevant representation for each auxiliary Young diagram.
} 
Now consider the simple example $F=2, m=2$, with symmetry group $U(1)$. (Recall that with $U(1)$ symmetry, both auxiliary diagrams yield only one representation, Ш.) The full spectrum has precisely one center of mass ground state, coming from the $\square$ auxiliary diagram for the $B$ operators.

In the product spectrum, we again represent each state by the corresponding auxiliary diagram. The center of mass annihilation operator acts as follows:

$$
a_{\mathrm{com}} \square=\square
$$

and also

$$
a_{\mathrm{com}} \boxminus=\square .
$$

Hence the product state $\square-$ 日 contains no center of mass energy.

On both sides, we have found the same number (1) of c.o.m. ground states, as expected in the $U(1)$ case where the spectra should match exactly. But the linear combination of product-spectrum auxiliary diagrams differs from that for the $B$ operators (口) and also from that of the original $A$ operators $\left[\square-\frac{1}{2} \boxminus\right.$, since $\left.\operatorname{Tr} B^{2}=\operatorname{Tr} A^{2}-\frac{1}{m}(\operatorname{Tr} A)^{2}\right]$ of the full spectrum.

One feature of this example turns out to generalize to arbitrary $F$ and $m$ (a proof is given in Appendix D.2): Each c.o.m. ground state in the product spectrum is a linear combination containing precisely one auxiliary diagram whose first two rows have equal length. These are the diagrams conjugate to the auxiliary diagrams defining the c.o.m. ground states of the full spectrum, which demonstrates that conjugation of Young diagrams does play a role in the mapping.

However, we conclude from the above example that even for the simple case of a $U(1)$ symmetry group, where the number of states manifestly agree on both sides, an individual product state is generically mapped into a linear combination of states in the natural basis for the full spectrum. It would be nice to understand this nontrivial mapping better.

\section{M-theory limit}

So far we have been working in the limit $\mu \rightarrow \infty$ at fixed arbitrary $N$. The M-theory limit corresponds to taking $N \rightarrow \infty$ at fixed $\mu$ and fixed longitudinal momentum $N / R$. We have focussed on vacua with $m$ coincident membranes, corresponding to the partition $\left(n^{m}\right)$. In this case the M-theory limit is $n \rightarrow \infty$ while holding $m$ and $n / R$ fixed. 
As mentioned in Sec. 2.2, the effective coupling in the coincident membrane configuration is

$$
\lambda=\left(\frac{R}{\mu n}\right)^{3} m,
$$

which is fixed in the M-theory limit, suggesting that perturbation theory remains valid [5].

On the other hand, Ref. [5] identified an explicit path leading to a change in the membrane configuration, with barrier height of order

$$
\frac{1}{n}\left(\frac{\mu n}{R}\right)^{3}
$$

(A membrane following this path extends a spike inward to its center and grows a small new spherical membrane there.) This barrier height vanishes like $1 / n$ in the M-theory limit, suggesting that perturbation theory breaks down.

This apparent paradox prompted the speculation [5] that the low energy decay path is very narrow in the space of perturbations about the minimum, and that thus perturbation theory is valid for generic perturbations up to much higher energies than (7.2).

Here we note that $\mu$ is no longer a physical parameter after $N \rightarrow \infty$ has been taken. It can be set to any value by a change of coordinates. Hence the question of whether or not a vacuum is perturbative in the M-theory limit cannot depend on the value of $\mu$. But taking the M-theory limit at small $\mu$ suggests that perturbation theory is completely invalid even for generic perturbations. This apparently conflicts with the expectation of a perturbative regime if we first take the M-theory limit at large $\mu$ and then rescale coordinates.

We expect that these two pictures are reconciled as follows. In the M-theory limit at large $\mu$ there is a small but finite decay rate for any vacuum at any finite temperature $T>0$. It is suppressed not by an energy barrier but by the time it takes to find the low pass through a high mountain range. Physically the coordinate rescaling of $\mu$ corresponds to a boost, and rescaling from very large to small $\mu$ requires an enormous boost, or more precisely, an undoing of the original boost that made $\mu$ large. The unboosted observer will find that all processes happen at a much higher rate, so the decay and mixing of vacua will be seen to occur instantaneously.

We hope to return to this question in more detail in future work.

\section{Acknowledgments}

We are grateful to T. Banks, D. Berenstein, P. Hořava, C. Keeler, J. Maldacena, A. Pasqua, S. Shenker, W. Taylor, and B. Tweedie for helpful discussions and com- 
ments. We are especially indebted to M. Aganagic and M. Van Raamsdonk for detailed explanations.

\section{A. Coefficients of irreducible representations}

In this appendix we list explicit results of a comparison between the full spectrum and the product spectrum. We have carried out this check for values up to and including $F=23$. The computational complexity and memory requirements grow exponentially with $F$. In all cases we have found that the product states form a subset of the full set of representations. This shows up in the table below in that all multiplicities of representations in the difference are positive.

Due to space constraints we list explicit results only for $6 \leq F \leq 10$ and all relevant $m$. The comparison for smaller $F$ was already carried out in Sec. 5.4. As explained above, for $m=1$ and $m \geq F$, the irreps on both sides match exactly, giving no net difference for these values of $m$. Therefore, in the table below, we do not go beyond $m=F-1$ (but include $m=1$ for completeness).

\begin{tabular}{|c|c|c|}
\hline$F$ & $m$ & $S^{\prime \prime}(m)_{F}-S^{\operatorname{prod}}(m)_{F}$ \\
\hline \multirow[t]{5}{*}{ 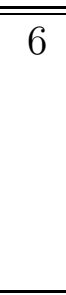 } & 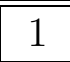 & - \\
\hline & 2 & $2(42)+(321)+2\left(2^{3}\right)$ \\
\hline & 3 & $2(42)+(321)+\left(3^{2}\right)+\left(2^{3}\right)$ \\
\hline & 4 & $(42)$ \\
\hline & 5 & - \\
\hline \multirow[t]{6}{*}{7} & 1 & - \\
\hline & 2 & $2(52)+(43)+2(421)+2\left(32^{2}\right)+\left(2^{3} 1\right)$ \\
\hline & 3 & $3(52)+3(43)+3(421)+\left(3^{2} 1\right)+3\left(32^{2}\right)+\left(2^{3} 1\right)$ \\
\hline & 4 & $2(52)+2(43)+\left(\begin{array}{lll}4 & 2 & 1\end{array}\right)+\left(32^{2}\right)$ \\
\hline & 5 & $(52)$ \\
\hline & 6 & - \\
\hline \multirow[t]{6}{*}{8} & 1 & - \\
\hline & 2 & $3(62)+(53)+2(521)+2\left(4^{2}\right)+(431)+4\left(42^{2}\right)+\left(32^{2} 1\right)+\left(2^{4}\right)$ \\
\hline & 3 & $\begin{array}{l}5(62)+5(53)+5(521)+3\left(4^{2}\right)+5(431)+7\left(42^{2}\right)+\left(421^{2}\right)+2\left(3^{2} 2\right)+ \\
2\left(32^{2} 1\right)+2\left(2^{4}\right)\end{array}$ \\
\hline & 4 & $4(62)+4(53)+3(521)+4\left(4^{2}\right)+3(431)+4\left(42^{2}\right)+\left(3^{2} 2\right)+\left(32^{2} 1\right)+\left(2^{4}\right)$ \\
\hline & 5 & 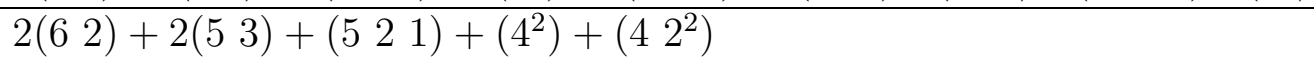 \\
\hline & & continued on next page \\
\hline
\end{tabular}




\begin{tabular}{|c|c|c|}
\hline & \multicolumn{2}{|c|}{ continued from previous page } \\
\hline & 6 & $(62)$ \\
\hline & 7 & - \\
\hline \multirow[t]{8}{*}{9} & 1 & - \\
\hline & 2 & $\begin{array}{l}3(72)+2(63)+3(621)+2(54)+\left(\begin{array}{ll}53 & 1\end{array}\right)+4\left(52^{2}\right)+2\left(4^{2} 1\right)+2(432)+ \\
2\left(42^{2} 1\right)+2\left(32^{3}\right)+\left(2^{4} 1\right)\end{array}$ \\
\hline & 3 & 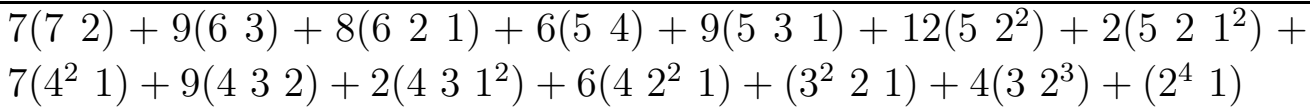 \\
\hline & 4 & 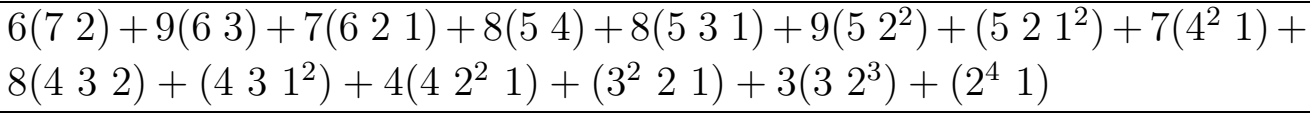 \\
\hline & 5 & 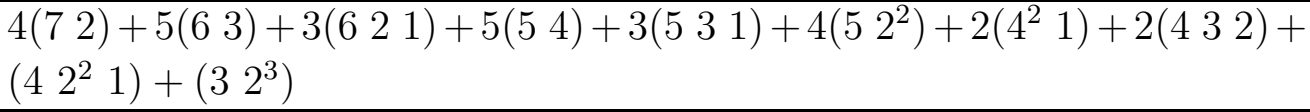 \\
\hline & 6 & 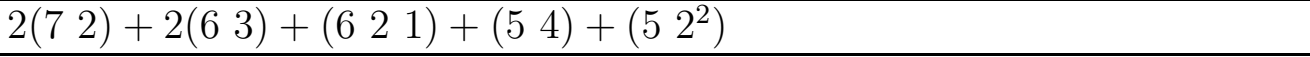 \\
\hline & 7 & $(72)$ \\
\hline & 8 & - \\
\hline \multirow[t]{9}{*}{10} & $\overline{11}$ & - \\
\hline & 2 & 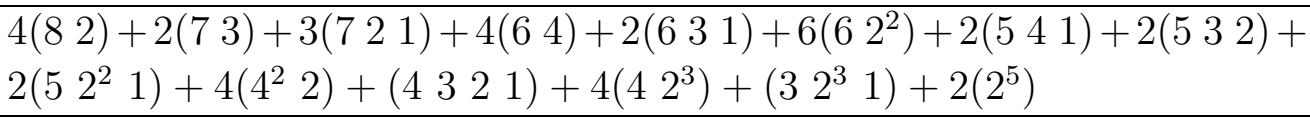 \\
\hline & 3 & 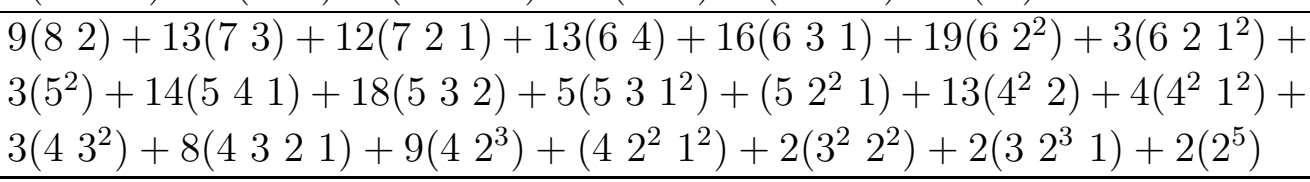 \\
\hline & 4 & $\begin{array}{l}10(82)+15(73)+12(721)+19(64)+19(631)+19\left(62^{2}\right)+3\left(621^{2}\right)+ \\
4\left(5^{2}\right)+18(541)+20(532)+5\left(531^{2}\right)+10\left(52^{2} 1\right)+16\left(4^{2} 2\right)+3\left(4^{2} 1^{2}\right)+ \\
4\left(43^{2}\right)+8(4321)+9\left(42^{3}\right)+\left(42^{2} 1^{2}\right)+2\left(3^{2} 2^{2}\right)+2\left(32^{3} 1\right)+2\left(2^{5}\right)\end{array}$ \\
\hline & 5 & 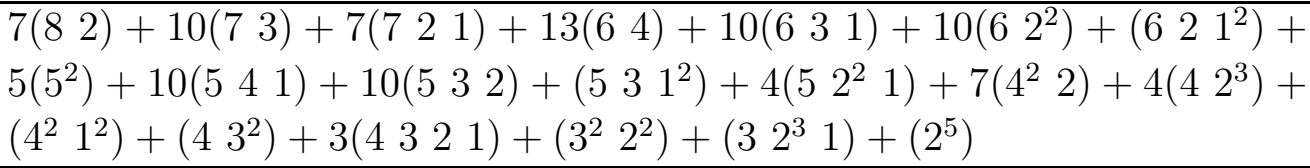 \\
\hline & 6 & 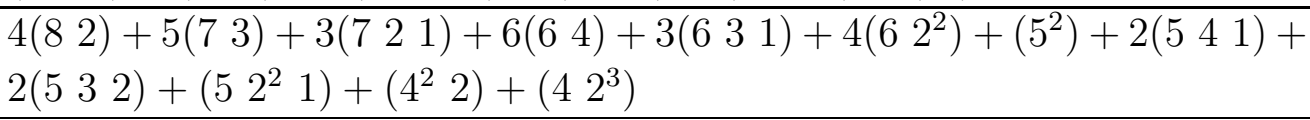 \\
\hline & 7 & $2(82)+2(73)+(721)+(64)+\left(62^{2}\right)$ \\
\hline & 8 & $(82)$ \\
\hline & 9 & - \\
\hline
\end{tabular}

\section{B. Representations and plethysm: Notation, examples, and properties}

In this appendix we summarize various definitions and properties concerning group 
representations and operations between them. Further details and references can be found, e.g., in Ref. [12].

\section{B.1 Young diagrams and tableaux}

The irreducible tensor representations of $U(m)$ with $r$ indices correspond to all possible ways of (anti-)symmetrizing the $r$-th power of the fundamental representation. Hence, they are in one-to-one correspondence with the representations of the symmetric group, and they can be denoted by Young diagrams with $r$ boxes and up to $m$ rows. These diagrams also label the partitions of $r$ with up to $m$ positive integer terms, where each row represents one term. ${ }^{16}$

We denote an irreducible representation $A$ by its Young diagram, (e.g., $A=\mathbb{\boxplus}^{\square}$ ) or by the corresponding partition of $r$, in the standard notation where "exponents" denote repeated terms (e.g., $A=\left(42^{2}\right)$ ). We denote the sum of two representations by $A \oplus B$ and the tensor product by $A \cdot B$. For $A \cdot A$ we also write $A^{2}$. For example, in $U(3)$,

$$
\theta^{2} \equiv 日 \cdot 日=\boxminus \oplus \boxplus
$$

A Young tableau is a Young diagram filled out with positive integers no larger than $m$, such that the numbers in each row are nondecreasing from left to right, and the numbers in each column are increasing from top to bottom. For a given Young diagram, the number of such tableaux is given by the usual "hook rule" and is equal to the dimension of the corresponding representation. (The requirements cannot be satisfied for diagrams with more than $m$ rows, which is why they have no states and can be ignored.)

\section{B.2 Schur functions}

Another way to describe an irreducible representation $A$ is to write down its Schur function $s_{A}$. The Schur function is the character (i.e., the trace) of the matrix representing a group element, as a function of (the conjugacy class of) the group element. It is a function of $m$ variables.

The Schur function is easily be obtained from the Young diagram as follows. For each allowed tableau, write down a product of $x$ 's indexed with the numbers appearing in the tableau; then add them all up. This will produce a symmetric polynomial of degree $r$ with positive coefficients whose sum is the dimension. For example, for $U(3)$, the representation $\boxplus$ (dimension 8) has the Schur function

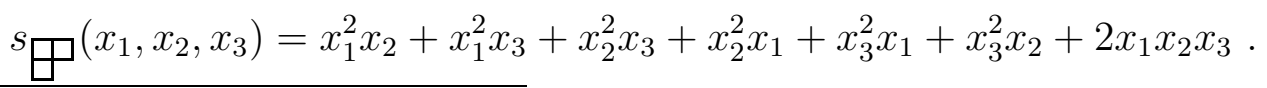

\footnotetext{
${ }^{16}$ Alternatively, these diagrams also label the partitions with no term greater than $m$, where each column represents one term.
} 
Schur functions of irreducible representations form a basis for the symmetric polynomials. The Schur functions of a reducible representation is the sum of the Schur functions of the irreducible representations it contains. One way to decompose the product of two representations is to multiply their Schur functions and expand the result in the basis of Schur functions of the appropriate degree. This reproduces the usual Littlewood-Richardson rule. For example, in $U(3)$,

$$
\begin{aligned}
\text { 日· } & =\left(x_{1} x_{2}+x_{1} x_{3}+x_{2} x_{3}\right)\left(x_{1}+x_{2}+x_{3}\right) \\
& =x_{1}^{2} x_{2}+x_{1}^{2} x_{3}+x_{2}^{2} x_{3}+x_{2}^{2} x_{1}+x_{3}^{2} x_{1}+x_{3}^{2} x_{2}+3 x_{1} x_{2} x_{3} \\
& =\boxminus \oplus 日 .
\end{aligned}
$$

If we are interested only in the final sum of diagrams, this computation can be carried out for the lowest value of $m$ for which all resulting representations exist (definitely $m$ need not be larger than the total number of boxes). The result will hold for all $m$; for small $m$, diagrams with more than $m$ boxes can be deleted.

\section{B.3 Plethysm}

Definition Plethysm is the composition of two Schur functions, denoted as $A^{\otimes B}$. Let $A$ be a representation of $U(m)$, and let $B$ be an arbitrary representation of $U(\operatorname{dim} A)$. (Neither $A$ nor $B$ are assumed irreducible.) Write the Schur function for $A$ as $s_{A}=$

$\sum_{i=1}^{\operatorname{dim} A} y_{i}$, where $y_{i}$ are the monomials arising from the individual Young tableaux. Plethysm of $A$ by $B$ is defined as

$$
A^{\otimes B} \equiv s_{B}(y)
$$

For example, for the $U(3)$ representation $A=$ 日 one has $y_{1}=x_{1} x_{2}, y_{2}=x_{1} x_{3}$, and $y_{3}=x_{2} x_{3}$. Let us take $B=$ 日. Then

$$
\begin{aligned}
\mathrm{\Theta}^{\otimes} \mathrm{\Theta} & =y_{1} y_{2}+y_{1} y_{3}+y_{2} y_{3} \\
& =x_{1}^{2} x_{2} x_{3}+x_{3}^{2} x_{1} x_{2}+x_{2}^{2} x_{1} x_{3} \\
& =\boxminus .
\end{aligned}
$$

Properties Plethysm has a number of properties that are used in this paper. It is clear from the definition that plethysm is right distributive, i.e., distributive in $B$ :

$$
A^{\otimes\left(B_{1} \oplus B_{2}\right)}=A^{B_{1}} \oplus A^{B_{2}} .
$$

It is also associative:

$$
\left(A^{\otimes B}\right)^{\otimes C}=A^{\otimes\left(B^{\otimes C}\right)} .
$$


The $g$-th tensor power of $A$ can be written as the sum over all plethysms of $A$ by Young diagrams with $g$ boxes:

$$
A^{g}=\bigoplus_{P(g)} A^{\otimes P}
$$

where the sum is over all partitions of $g$. [Comparison of Eqs. (B.5) and (B.10) with Eq. (B.1) will give an example of this for $A=\boxminus$ and $g=2$.]

Special cases In this paper we frequently consider two special cases of plethysm which get their own name. The first is $B=(g)$, i.e., the Young diagram with one row, corresponding to the totally symmetric representation with $g$ indices. We denote plethysm by $(g)$ as sym $A^{g}$ :

$$
\text { sym } A^{g} \equiv A^{(g)} .
$$

E.g., for the $U(3)$ representation $A=\mathrm{日}$

$$
\begin{aligned}
\operatorname{sym} A^{2} \equiv \Xi^{\otimes \square} & =y_{1}^{2}+y_{2}^{2}+y_{3}^{2}+y_{1} y_{2}+y_{1} y_{3}+y_{2} y_{3} \\
& =x_{1}^{2} x_{2}^{2}+x_{1}^{2} x_{3}^{2}+x_{2}^{2} x_{3}^{2}+x_{1}^{2} x_{2} x_{3}+x_{3}^{2} x_{1} x_{2}+x_{2}^{2} x_{1} x_{3} \\
& =\boxplus .
\end{aligned}
$$

The second important case is $B=\left(\right.$ oddrow $\left._{g}\right)$, i.e., the sum of all Young diagrams with $g$ boxes and an odd number of (nonzero) rows. We denote plethysm by (oddrow ${ }_{g}$ ) as cyc $A^{g}$ :

$$
\text { cyc } A^{g} \equiv A^{\left.\otimes \text { (oddrow }_{g}\right)} .
$$

E.g., for the $U(3)$ representation $A=\mathrm{日}$

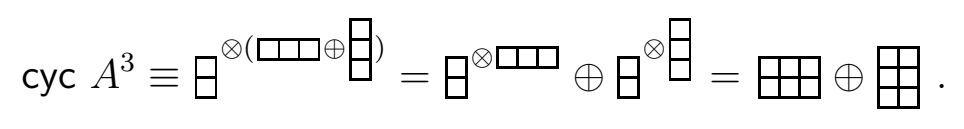

Interpretation Plethysm is interpreted as follows. Think of $B$ as a representation of the permutation group, and let $g$ be the number of boxes in the Young diagram of $B$. Plethysm of $A$ by $B$ computes the restriction of the $g$-th tensor power of $A$ to the symmetrization pattern $B$.

Let us discuss this in more detail for the two types of plethysm mainly considered in this paper. Suppose $A$ represents a $U(m)$ multiplet of creation operators (i.e., $A$ carries $U(m)$ indices but it is not a matrix). If all creation operators commute, the set of states created by $A A A|0\rangle$ will form the representations computed by sym $A^{3} \equiv A^{\otimes} \square$, and not by $A^{3}$, since exchange of the complete set of indices between different $A$ 's gives the same state. 
The cyc plethysm defined in Eq. (B.11) computes the representations formed by the trace of a product of $g$ matrices of rank $h \geq g$ :

$$
\operatorname{Tr} A A A \ldots A|0\rangle \text {, }
$$

where the matrix $A$ represents a $U(m)$ multiplet of matrix operators.

To see this, note that the correct plethysm $B$ will be the sum of all $g$-box Young diagrams $B_{\alpha}$ whose symmetrization pattern is compatible with the manifest invariance of Eq. (B.13) under cyclic exchange of indices:

$$
B=\sum_{\alpha} B_{\alpha} .
$$

It remains to show that a $g$-box Young diagram satisfies this criterion if and only if it has an odd number of rows.

Consider filling a given Young diagram $B_{\alpha}$ with integers $1 \ldots g$ from left to right, top to bottom row. An elementary cyclic permutation produces a different Young tableau by shifting every integer to the right, and the integer at the end of each row to the beginning of the next row; $g$ now occupies the top left box. Invariance under cyclic permutations requires that these two Young tableaux appear with the same sign in the symmetrization pattern $B_{\alpha}$. Recall that this consists of first totally symmetrizing within each row, then totally antisymmetrizing over the indices appearing within each column. Starting from the first Young tableau, we rearrange the entries in each row so that they agree with the second Young tableau everywhere except in the first column. Since this involves only manipulations within rows we pick up no minus sign. To end up with the second tableau, we now need to move all entries in the first row down by one box (and the $g$ up to the top left box). If $B_{\alpha}$ has $q$ rows, this will involve $q-1$ pairwise permutations. Each contributes a factor $(-1)$, so the overall sign is $(-1)^{q-1}$, which is positive precisely if $q$ is odd. This shows that odd $q$ is necessary. It is also sufficient, since all other cyclic permutations are powers of the elementary one we considered.

\section{Trace relations}

In this appendix we discuss the efficacy of the truncation in Eq. (3.9) in eliminating redundant states. We consider a general case with $k$ different matrices of size $m \times m$. In the context of this paper, $k=\operatorname{dim}\left(R(n)_{1}\right)$; see Eq. (3.6).

The case of only a single species, $k=1$, is well understood. Taking into account gauge invariance under $U(m)$, there are $m$ physical degrees of freedom. Single trace states $\operatorname{Tr}\left(A^{i}\right)|0\rangle$ of length up to $m$ are mutually independent. For $i>m$ the trace is always equal to a product of shorter traces. All independent multitrace states are thus constructed from single traces of length up to $m$. 


\section{C.1 Algebraic relations}

For more than one species of matrix creation operator, $k>1$, the situation is more complicated. ${ }^{17}$ Gauge invariance allows the elimination of up to $m^{2}$ gauge degrees of freedom, leaving $(k-1) m^{2}$ physical degrees of freedom. But there are at least $k^{F} / F$ single traces of length $F$ that can be written down, (the division by $F$ taking into account the cyclic identity of the trace). This number grows quickly with $F$. It is easy to see that for large enough $m,(k-1) m^{2}<k^{F} / F$ for values of $F$ at least of order $\frac{\log m}{\log k}$, which is much smaller than $m$.

Let us imagine for a moment that the matrices consist not of operators but of numbers. Taking a trace does not increase the number of independent parameters, so most of the traces of length greater than $\frac{\log m}{\log k}$ must be algebraically related. This means that most traces of length up to $m$ (which can be much larger) are not algebraically independent of each other.

However, this argument does not carry over straightforwardly to matrix operators. Even simple algebraic relations may involve, e.g., some square roots, and thus will not hold between states obtained from matrix operators acting on the vacuum. Hence, the above counting argument does not automatically imply that the single-trace states of length up to $m$ fail to be independent [13]. (In fact we will argue below that they are indeed independent.)

On the other hand, it may be possible to convert some algebraic relations into linear relations between polynomials of single traces. This would correspond to trace relations between certain multitrace states. In fact, it is possible to construct an explicit example (with $m=2, F=10, k \geq 5$ ) of an identity relating apparently different multitrace states, each of which involves only products of traces of length up to $m$ [13]. This demonstrates that not all the states we consider in the spectrum $R^{\prime}$ are independent: we are overcounting states and representations in $R^{\prime}$.

We will now argue, however, that the spectrum $R^{\prime}$ is an excellent approximation to the true spectrum for values of $F \ll k m^{2}$. (Recall that the total occupation number $F$ is the number of matrix creation operators acting on the vacuum.) Note that $k$ is of order 10 already for $n=1$ (one unit of momentum per fuzzy sphere), grows quadratically with $n$, and diverges in the M-theory limit.

\section{C.2 Thermodynamic limit}

We begin with an argument explaining this scale. For $m \rightarrow \infty$, there are no trace relations. In this case the number of single trace states really is at least $k^{F} / F$, which gives a Hagedorn-like spectrum. The same will hold for multitrace states of total length $F$

\footnotetext{
${ }^{17}$ The following argument was explained to us by M. Van Raamsdonk [13].
} 
since the entropy contribution from partitioning $L$ into individual traces is subleading. As pointed out in Ref. [14], this behavior cannot persist for finite $m$ at energies $E \approx F$ much greater than $\mathrm{km}^{2}$, because it would contradict the thermodynamics of a finite quantum mechanical system with $\mathrm{km}^{2}$ degrees of freedom.

The thermodynamic treatment becomes valid when the temperature is at least of order unity. Then $E \sim k m^{2} T$ and $S \sim k m^{2} \log T$. Thus, for $E>k m^{2}$, the entropy should grow only logarithmically with the total occupation number:

$$
S \sim k m^{2} \log \frac{E}{k m^{2}} .
$$

The only natural explanation for the discrepancy with the case of infinite matrices, of course, is the appearance of trace relations for finite matrices. Hence, trace relations must become important at energies above $F \sim k m^{2}$. Below this energy scale the finiteness of the number of degrees of freedom should be difficult to detect. The system should behave display the Hagedorn-like behavior of infinite matrices. Hence, one expects trace relations to have a negligible influence on the spectrum for $F \ll k m^{2}$.

\section{C.3 Exact number of states: A bosonic model}

Next, we derive a formula for the precise number of states, fully taking into account trace relations. We will find that no trace relations arise for total occupation number $F \leq m$, justifying our assumption that single trace states of length up to $m$ are mutually independent. For larger energies, we will compare our result to the number of states in the approximation we employ in Sec. 3.2 (obtained by limiting the length of individual traces to $m$ and ignoring trace relations). In the regime where we can evaluate our two expressions explicitly, their agreement will turn out to be excellent, with no evidence for a significant effect from trace relations.

We will carry this out for a purely bosonic model but we expect that the trace relations will have the same (small) effect when fermions are included.

\section{C.3.1 Exact number of states of the full spectrum}

Consider a theory with $k$ bosonic matrix creation operators, each of which contributes unity to the total "energy" $F$. We are interested in the case where the operators transform in the adjoint of $U(m)$.

The exact number of states with occupation number $F, \mathcal{N}(F)$, is given by the coefficient of $x^{F}$ in the partition function, where $x=e^{-\beta} \cdot{ }^{18}$ The partition function is

\footnotetext{
${ }^{18}$ We parametrize the spectrum by total occupation number $F$, which is equal to the energy only if all operators contribute unit energy. In the plane wave model this is true only approximately. Here $\beta$ is conjugate to $F$ and thus is only approximately an inverse temperature.
} 
given by an integral over the group ${ }^{19} U(m)[14]$ :

$$
Z(x)=\int[d U] \exp \sum_{n=1}^{\infty} k x^{n} \frac{\operatorname{Tr}\left(U^{n}\right) \operatorname{Tr}\left(U^{\dagger n}\right)}{n} .
$$

Taylor expansion yields

$$
\begin{aligned}
Z(x) & =\int[d U] \prod_{n=1}^{\infty}\left(\sum_{q_{n}=0}^{\infty} x^{n q_{n}} \frac{\left[\left(\operatorname{Tr}\left(U^{n}\right) \operatorname{Tr}\left(U^{\dagger n}\right)\right]^{q_{n}}\right.}{n^{q_{n}} q_{n} !}\right)^{k} \\
& =\int[d U]\left(\sum_{\vec{q}} x^{|\vec{q}|} \frac{\operatorname{Tr}_{\vec{q}}(U) \operatorname{Tr}_{\vec{q}}\left(U^{\dagger}\right)}{\zeta(\vec{q})}\right)^{k}
\end{aligned}
$$

where the sum is over all (infinite-dimensional) vectors $\vec{q}=\left(q_{1}, q_{2}, \ldots\right)$ with nonnegative integer coefficients, and we define

$$
\begin{aligned}
|\vec{q}| & \equiv \sum_{n=1}^{\infty} n q_{n}, \\
\operatorname{Tr}_{\vec{q}}(U) & \equiv \prod_{n=1}^{\infty}\left[\operatorname{Tr}\left(U^{n}\right)\right]^{q_{n}} \\
\zeta(\vec{q}) & \equiv \prod_{n=0}^{\infty} n^{q_{n}} q_{n} !
\end{aligned}
$$

This can be further expanded to

$$
Z(x)=\int[d U] \sum_{\vec{q}^{(1)}} \cdots \sum_{\vec{q}^{(k)}} x^{|\vec{Q}|} \frac{\operatorname{Tr}_{\vec{Q}}(U) \operatorname{Tr}_{\vec{Q}}\left(U^{\dagger}\right)}{\prod_{i=1}^{k} \zeta\left(\vec{q}^{(i)}\right)}
$$

where $\vec{Q}$ is the sum of the $k$ vectors $\vec{q}^{(1)}, \ldots, \vec{q}^{(k)}$ :

$$
\vec{Q} \equiv \sum_{i=1}^{k} \vec{q}^{(i)}
$$

To extract the degeneracy at occupation number $F$, we restrict the above sum to $|\vec{Q}|=F$ :

$$
\mathcal{N}(F)=\int[d U] \sum_{\vec{q}^{(i)}:|\vec{Q}|=F} \frac{\operatorname{Tr}_{\vec{Q}}(U) \operatorname{Tr}_{\vec{Q}}\left(U^{\dagger}\right)}{\prod_{i=1}^{k} \zeta\left(\vec{q}^{(i)}\right)} .
$$

\footnotetext{
${ }^{19}$ We thank M. Van Raamsdonk for suggesting this method of evaluating the spectrum.
} 
Only the denominator depends on the detailed decomposition of $\vec{Q}$ into $k$ vectors. This part of the sum can be evaluated using the identity

$$
\sum_{\vec{q}^{(i)}: \vec{Q} \text { fixed }} \frac{\zeta(\vec{Q})}{\prod_{i=1}^{k} \zeta\left(\vec{q}^{(i)}\right)}=k^{\|\vec{Q}\|}
$$

where

$$
\|\vec{Q}\| \equiv \sum_{n=1}^{\infty} Q_{n}
$$

This yields

$$
\mathcal{N}(F)=\int[d U] \sum_{\vec{Q}:|\vec{Q}|=F} k^{\|\vec{Q}\|} \frac{\operatorname{Tr}_{\vec{Q}}(U) \operatorname{Tr}_{\vec{Q}}\left(U^{\dagger}\right)}{\zeta(\vec{Q})}
$$

The integral can be evaluated as follows. Each conjugacy class of the symmetric group $S_{h}$ corresponds to a vector $\vec{q}$ with $|\vec{q}|=h$. (One can view $\vec{q}$ as a Young diagram. The component $q_{i}$ is the number of $i$-cycles; the number of elements in the conjugacy class is $\zeta(\vec{q})$.) The group characters $\chi$ in any two irreducible representations $R_{1}$ and $R_{2}$ with $h$ boxes satisfy the orthogonality relations

$$
\begin{aligned}
& \sum_{\vec{q}} \frac{\chi_{R_{1}}(\vec{q}) \chi_{R_{2}}(\vec{q})}{\zeta(\vec{q})}=\delta_{R_{1} R_{2}} \\
& \sum_{R} \frac{\chi_{R}(\vec{q}) \chi_{R}\left(\vec{q}^{\prime}\right)}{\zeta(\vec{q})}=\delta_{\vec{q} \vec{q}^{\prime}} .
\end{aligned}
$$

In both cases the sum runs over all Young diagrams with $h$ boxes.

Similarly, the characters of the unitary group $U(m)$ satisfy

$$
\int[d U] \operatorname{Tr}_{R_{1}} U \operatorname{Tr}_{R_{2}} U^{\dagger}=\delta_{R_{1} R_{2}}
$$

where $R_{1}$ and $R_{2}$ are arbitrary irreducible representations of $U(m)$, and $\operatorname{Tr}_{R} U$ is the trace of the group element $U$ in the representation $R$. Recall that for finite $m$, the irreducible representations of $U(m)$ are in one-to-one correspondence with Young diagrams with up to $m$ rows.

Using these relations and the Frobenius formula it can be shown that [15]

$$
\operatorname{Tr}_{\vec{q}} U=\sum_{R} \chi_{R}(\vec{q}) \operatorname{Tr}_{R} U
$$

The sum runs over all diagrams with $|\vec{q}|$ boxes, but for finite $m$, only Young diagrams with up to $m$ rows will contribute since the remaining representations do not exist. It 
follows that

$$
\int[d U] \operatorname{Tr}_{\vec{q}} U \operatorname{Tr}_{\vec{q}^{\prime}} U^{\dagger}=\sum_{R} \chi_{R}(\vec{q}) \chi_{R}\left(\vec{q}^{\prime}\right) .
$$

Thus the total number of states with occupation number $F$ is

$$
\mathcal{N}(F)=\sum_{\substack{\vec{Q}:|\vec{Q}|=F \\ R: b(R)=F, r(R) \leq m}}\left[\chi_{R}(\vec{Q})\right]^{2} \frac{k^{\|\vec{Q}\|}}{\zeta(\vec{Q})},
$$

where $b(R)$ and $r(R)$ denote the number of boxes and rows of $R$.

Note that for $F \leq m$, the restriction on the number of rows is trivial and the number of states becomes independent of $m$ in this regime. This includes the limit of infinitely large matrices, in which there are no trace relations. Hence there will be no trace relations for any value of $m \geq F$, as advertised earlier.

As a consistency check, for $F \leq m$, Eq. (C.19) can be evaluated using the identity [15]

$$
\int[d U] \operatorname{Tr}_{\vec{q}} U \operatorname{Tr}_{\vec{q}^{\prime}} U^{\dagger}=\delta_{\vec{q} \vec{q}^{\prime}} \zeta(\vec{q})
$$

This yields

$$
\mathcal{N}(F)=\sum_{\vec{Q}:|\vec{Q}|=F} k^{\|\vec{Q}\|},
$$

which agrees with the result obtained by expanding the $m \rightarrow \infty$ partition function [14]

$$
Z_{\infty}(x)=\prod_{n=1}^{\infty} \frac{1}{1-k x^{n}}
$$

\section{C.3.2 Exact number of states ignoring trace relations}

For $F \geq m$, the exact number of states, Eq. (C.19), will be smaller than the $m \rightarrow \infty$ value, Eq. (C.21), reflecting the redundancy of states due to trace relations at finite $m$. As explained in Sec. C.2 above, however, we expect the approximation in Sec. 3.2 to be quite accurate as long as the energy does not become too large: $F \ll \mathrm{km}^{2}$. This approximation consisted of ignoring trace relations (which is tantamount to taking the size of the matrix to infinity) and instead removing all states containing single traces of length greater than $m$. (Please note that $m$ no longer corresponds to the size of the matrix in the analysis below!)

The exact number of states in this approximation can be obtained from a tedious but straightforward generalization of results presented in Ref. [14]. (Their most nontrivial aspect is the use of Polya theory to take the cyclic symmetry of the trace into 
account.) The partition function is given by

$$
Z_{\text {ap }}(x)=\exp \sum_{\eta=1}^{\infty} \psi_{k m}(\eta) \frac{x^{\eta}}{\eta}
$$

where

$$
\psi_{k m}(\eta)=\sum_{\sigma \mid \eta=1}^{m} \sum_{q \mid \sigma} \phi(q) K^{\sigma / q}
$$

and $\phi(q)$ is the number of coprimes of $q$ which are not larger than $q$. Notation such as $q \mid \sigma$ denotes integer divisors $q$ of $\sigma$.

The number of states with total operator number $F$ is obtained by expanding and extracting the coefficient of $x^{F}$ :

$$
\mathcal{N}_{\text {ap }}(F)=\sum_{\vec{q}:|\vec{q}|=F} \frac{\prod_{\eta=1}^{\infty}\left[\psi_{k m}(\eta)\right]^{q_{\eta}}}{\zeta(\vec{q})}
$$

Fig. If shows a comparison of $\mathcal{N}$ and $\mathcal{N}_{\text {ap }}$ for some sample values of parameters. We were able to compute ${ }^{20}$ both expressions for all values of $m$ in the range $K \leq 100$, $F \leq 7$. We found that the agreement is very good. In all examples, our approximation was either exactly correct $\left(\mathcal{N}_{\text {ap }}(F)=\mathcal{N}(F)\right)$ or slightly smaller than the true number of states. This is consistent with our expectation that a small number of single traces of length greater than $m$ do contribute to independent states (but they are discarded in our approximation, giving a small undercount), while the trace relations ignored in our approximation eliminate only a tiny or vanishing number of states (giving a tiny overcount).

In concluding this appendix, two more remarks are in order. First, it is possible that both the under- and overcount are quite large but happen to cancel almost precisely. In this case our approximation would not be give a reliable set of representations.

Second, we have computed $\mathcal{N}$ only in the the regime $F \lesssim k m^{2}$. Thus our results are consistent with the earlier thermodynamic argument suggesting that trace relations should be negligible below $F \sim k m^{2}$. However, we were unable so far to compute $\mathcal{N}(F)$ for any $F>7$, so our numerical work alone cannot pin down the onset of trace relations (which could be already around $F \sim k m$, for example, without being diagnosed by our numerical results). It seems likely that our method of computing $\mathcal{N}(F)$ can be significantly improved to achieve higher speeds and larger $F$.

\footnotetext{
${ }^{20}$ We thank M. Marino for CHAR, a Mathematica routine computing characters of symmetric groups.
} 

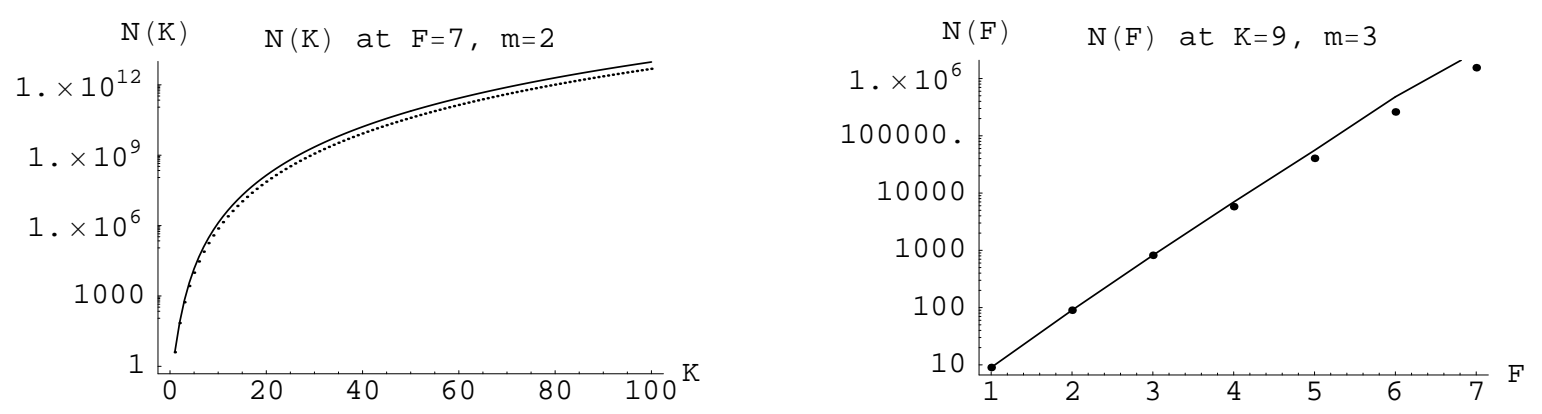

Figure 1: The solid line shows the exact number of states $\mathcal{N}$, taking into account trace relations. The dots shows the number of states $\mathcal{N}_{\text {ap }}$ resulting from the approximation employed in the main part of the paper (limiting single traces to length $m$ but otherwise ignoring potential trace relations).

\section{Proofs}

This Appendix contains proofs of two statements made in the main text.

\section{D.1 Distinguishable particles}

Here we prove the inclusion relation Eq. (3.10) for a pair of toy spectra in which traces and particles are treated as distinguishable. That is, we drop the outer symmetrization requirement in Eqs. (5.11) and (5.12). The corresponding spectra are

$$
\begin{aligned}
& T^{\prime \prime}\left(n^{m}\right)_{F}=\bigoplus_{P(F \backslash m)=\left(q_{1}^{\left.p_{1} \ldots q_{l}^{p_{l}}\right)}\right.} \prod_{i=1}^{l}\left(\operatorname{sym} R(n)_{1}^{q_{i}}\right)^{p_{i}} \\
& T^{\text {prod }}\left(n^{m}\right)_{F}=\bigoplus_{P(F / m)=\left(q_{1}^{\left.p_{1} \ldots q_{l}^{p_{l}}\right)}\right.} \prod_{i=1}^{l}\left(\operatorname{sym} R(n)_{1}^{q_{i}}\right)^{p_{i}} \text {. }
\end{aligned}
$$

We will now show that $T^{\prime \prime}\left(n^{m}\right)_{F} \supseteq T^{\text {prod }}\left(n^{m}\right)_{F}$.

The auxiliary Young diagrams for these toy spectra are unaffected by this modification. Its only effect is to change the product of rows into a regular, rather than a symmetrized product, when the representations are computed.

We have already learned that auxiliary diagrams appearing in both spectra can be canceled. At every $m$ and $F$, the remaining diagrams will be related to each other by conjugation. All uncancelled diagrams in the full spectrum will be higher than wide, and vice-versa for the product spectrum. 
One can then deform each remaining auxiliary diagram of the full spectrum into its conjugate diagram (corresponding to one of the remaining auxiliary diagrams of the product spectrum) by a sequence of steps which strictly reduce the number of representations generated by the auxiliary diagram.

Each step takes one box and moves it to a higher row. Because there is no need to symmetrize rows, the effect of this move will be limited to the product between the origin row and the target row. Suppose the length of the origin row before the move is $l$ and the length of the target row is $k$. By construction, $k \geq l$. Originally the two rows contribute

$$
(k) \cdot(l)=(k+l) \oplus(k+l-1 \quad 1) \oplus \cdots \oplus(k+1 \quad l-1) \oplus\left(\begin{array}{ll}
k & l
\end{array}\right)
$$

to the product of rows. After moving the box, the contribution will be

$$
(k+1) \cdot(l-1)=(k+l) \oplus(k+l-1 \quad 1) \oplus \cdots \oplus(k+1 \quad l-1) .
$$

The latter is a subset of the former since the $(k l)$ representation is missing. By a sequence of such steps one arrives at the conjugate of the original Young diagram.

Thus each uncancelled auxiliary diagram in the full spectrum leads to representations which are a superset of those generated by the corresponding (conjugate) leftover diagram in the product spectrum.

\section{D.2 Mapping states with $U(1)$ spacetime symmetry}

Here we prove a result constraining the mapping between states with no center-ofmass excitation (see Sec. 6.2) in a toy theory with symmetry group $U(1)$ (see Sec. 6.1 for its simplifying properties, which allow an exact matching of spectra). Recall that the c.o.m. ground states in the full spectrum are in one-to-one correspondence with auxiliary Young diagrams whose first and second column are equally high. (This is true whether we think of this diagram as controlling traces of powers of $B$, or of $A$, as defined in Sec. 6.2.)

A blunt diagram is a Young diagram whose first and second row have equal length. Blunt diagrams are conjugate to the c.o.m. ground state diagrams in the full spectrum, and so we expect that they will play a special role in characterizing c.o.m. ground states of the product spectrum.

We prove that a basis of c.o.m. ground states in the product spectrum can be chosen so that each basis element is a linear combination containing precisely one blunt auxiliary diagram.

To prove this, let us first understand how the c.o.m. annihilation operator acts on an arbitrary diagram. Here is the list of diagrams with $F=5$, which get converted 
into sums of $F=4$ states.

$$
\begin{aligned}
& \text { पा } \rightarrow \text { माम }
\end{aligned}
$$

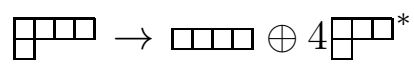

$$
\begin{aligned}
& \boxplus \rightarrow 2 \boxminus \oplus 3 \boxplus^{*} \\
& \Theta^{\square} \rightarrow \Theta^{\oplus} \oplus 3 \bigoplus^{*} \\
& \boxplus ! \rightarrow \boxplus \oplus 4 \boxplus \\
& \Theta \rightarrow \boxplus \oplus 2 \theta^{*} \\
& \text { 日 } ! \rightarrow \text { 日 }
\end{aligned}
$$

On the left are the $F=5$ "parent" diagrams on which $a_{\text {com }}$ acts; on the right are $F=4$ daughters. Note that the sums here are linear superpositions of states, not an addition of representations $(\oplus)$.

One finds these relations by working explicitly with operators, and it is straightforward to generalize them. The general rule is that the daughters are obtained by all legal ways to remove one box from a given parent. Each such way corresponds to one "block" in the parent diagram formed by rows of equal length. The coefficients of daughters are given by the number of boxes in the corresponding block on the left.

In the above list, we have marked the blunt parent diagrams by exclamation marks. On the right, we have added stars to certain daughter diagrams which have a special property that will be useful to us: each can be reverted back into its $F=5$ parent diagram by adding a box on the top right. Obviously none of the daughters of blunt parents have this property, and exactly one daughter of any non-blunt parents does.

To show that any blunt diagram can be combined with non-blunt diagrams to form a c.o.m. ground state, we need to cancel off the daughters. We give a general argument but it may be instructive to follow along using the above example. Add one box to the top right of each daughter of the blunt diagram. This will give us a nonblunt diagram that can be used to cancel every daughter of the blunt diagram, at the cost of introducing new daughters. However, none of these new daughters will carry a star (since the starred daughters were used to cancel the blunt diagram's daughters). Hence one can continue adding a box to the top right and canceling. This procedure always increases the number of columns but keeps the number of boxes fixed; hence it terminates. This completes the first part of our proof.

For the second part, we must show that a product state with no c.o.m. excitation cannot be obtained purely from non-blunt diagrams. Suppose the opposite and start 
with any non-blunt diagram. One of its daughters is starred and can only be canceled by daughters of another diagram obtained by adding a box to some row other than the first row. By assumption this diagram is non-blunt and hence will produce another starred daughter. Continuing in this manner, we never increase the length of the first row, and hence we must eventually be increasing the number of rows. This will eventually require a number of rows larger than $m$ which is not allowed, or for $m \geq F$ it will force us to use the one-column parent diagram, which is blunt. This contradicts our assumption, completing the proof.

\section{References}

[1] T. Banks, W. Fischler, S. H. Shenker and L. Susskind: M theory as a matrix model: A conjecture. Phys. Rev. D 55, 5112 (1997), hep-th/9610043.

[2] J. Maldacena: The large $N$ limit of superconformal field theories and supergravity. Adv. Theor. Math. Phys. 2, 231 (1998), hep-th/9711200.

[3] D. Berenstein, J. M. Maldacena and H. Nastase: Strings in flat space and pp waves from $N=4$ super Yang Mills. JHEP 04, 013 (2002), hep-th/0202021.

[4] W. Taylor: M(atrix) theory: Matrix quantum mechanics as a fundamental theory. Rev. Mod. Phys. 73, 419 (2001), hep-th/0101126.

[5] K. Dasgupta, M. M. Sheikh-Jabbari and M. Van Raamsdonk: Matrix perturbation theory for M-theory on a PP-wave. JHEP 05, 056 (2002), hep-th/0205185.

[6] K. Dasgupta, M. M. Sheikh-Jabbari and M. Van Raamsdonk: Protected multiplets of M-theory on a plane wave. JHEP 09, 021 (2002), hep-th/0207050.

[7] J. Maldacena, M. M. Sheikh-Jabbari and M. Van Raamsdonk: Transverse fivebranes in matrix theory. JHEP 01, 038 (2003), hep-th/0211139.

[8] L. Susskind: Another conjecture about M(atrix) theory (1997), hep-th/9704080.

[9] A. Baha Balantekin and I. Bars: Branching rules for the supergroup $S U(N / M)$ from those of $S U(N+M)$. J. Math. Phys. 23, 1239 (1982).

[10] O. Aharony, J. Sonnenschein and S. Yankielowicz: Interactions of strings and D-branes from $M$ theory. Nucl. Phys. B474, 309 (1996), hep-th/9603009.

[11] LiE: A computer algebra package for Lie group computations, http://wwwmathlabo.univ-poitiers.fr/ maavl/LiE/ .

[12] B. Fauser, P. Jarvis, R. King and B. Wybourne: New branching rules induced by plethysm (2005), math-ph/0505037. 
[13] M. Van Raamsdonk: unpublished.

[14] O. Aharony, J. Marsano, S. Minwalla, K. Papadodimas and M. Van Raamsdonk: The Hagedorn / deconfinement phase transition in weakly coupled large $N$ gauge theories. Adv. Theor. Math. Phys. 8, 603 (2004), hep-th/0310285.

[15] M. Aganagic, H. Ooguri, N. Saulina and C. Vafa: Black holes, q-deformed 2d Yang-Mills, and non-perturbative topological strings. Nucl. Phys. B715, 304 (2005), hep-th/0411280. 